\title{
A Model-free Four Component Scattering Power Decomposition for Polarimetric SAR Data
}

\author{
Subhadip Dey (D), Graduate student Member, IEEE, Avik Bhattacharya (D), Senior Member, IEEE, \\ Alejandro C. Frery (D), Senior Member, IEEE, and Carlos López-Martínez (D), Senior Member, IEEE, and \\ Yalamanchili S. Rao (D), Member, IEEE
}

\begin{abstract}
Target decomposition methods from polarimetric Synthetic Aperture Radar (PolSAR) data provides target scattering information. In this regard, several conventional model-based methods use scattering power components to analyze polarimetric SAR data. However, the typical hierarchical process to enumerate power components uses various branching conditions, leading to several limitations. These techniques assume ad hoc scattering models within a radar resolution cell. Therefore, the use of several models makes the computation of scattering powers ambiguous. Some common issues of model-based decompositions are related to the compensation of the orientation angle about the radar line of sight and the occurrence of negative power components. We propose a model-free four-component scattering power decomposition that alleviates these issues. In the proposed approach, we use the non-conventional 3D Barakat degree of polarization to obtain the polarization state of scattered electromagnetic wave. The degree of polarization is used to obtain the even-bounce, odd-bounce, and diffused scattering power components. Along with this, a measure of target scattering asymmetry is also proposed, which is then suitably utilized to obtain the helicity power. All the power components are rollinvariant, non-negative and unambiguous. In addition to this, we propose an unsupervised clustering technique that preserves the dominance of the scattering power components for different targets. This clustering technique assists in understanding the importance of diverse scattering mechanisms based on target characteristics. The technique adequately captures the clusters' variations from one target to another according to their physical and geometrical properties. In this study, we utilized L-, C-, and X-band full-polarimetric SAR data. We used these three data sets to show the effectiveness of decomposition powers and the natural interpretability of clustering results. The code is available at: https://github.com/Subho07/MF4CF
\end{abstract}

Index Terms-Full polarimetry, Synthetic aperture radar, Target decomposition, scattering-type parameter, Target characterization

\section{INTRODUCTION}

$\mathbf{P}$ OLARIMETRIC SAR decomposition methods based on coherency or covariance matrix representation either follow model-based or eigenvector analysis. On the one hand, eigenvector approaches determine a set of coherent scattering

S. Dey, A. Bhattacharya, and Yalamanchili S. Rao are with the Microwave Remote Sensing Lab, Center of Studies in Resources Engineering, Indian Institute of Technology Bombay, India (e-mail: sdey2307@gmail.com).

Alejandro C. Frery is with the School of Mathematics and Statistics, Victoria University of Wellington, New Zealand, and with the Key Lab of Intelligent Perception and Image Understanding of the Ministry of Education, Xidian University, Xi'an, China (e-mail: alejandro.frery@vuw.ac.nz).

Carlos López-Martínez is with the Signal Theory and Communications Department (TSC), Universitat Politécnica de Catalunya (UPC), Barcelona, Spain (e-mail: carlos.lopez@tsc.upc.du). mechanisms and describe the in-scene scatterers in an average sense. On the other hand, model-based decompositions first define and parameterize the set of canonical scatterers used to describe the scene. A combination of these canonical scatterers is used to generate a good fit for the polarimetric coherency or covariance matrix.

For incoherent targets, Freeman and Durden [1] proposed a three-component (i.e., surface, double-bounce, and volume) model-based decomposition technique based on the assumption of target reflection symmetry, i.e., $\left\langle S_{\mathrm{HH}} S_{\mathrm{HV}}^{*}\right\rangle=$ $\left\langle S_{\mathrm{VV}} S_{\mathrm{VH}}^{*}\right\rangle=0$. A cloud of randomly oriented dipoles is considered as the volume scattering model. The FreemanDurden 3-component scattering power technique, which is intuitive and easy to implement, has been widely used in numerous applications [2], [3].

However, the assumption of reflection symmetry is often limited to natural targets, e.g. forest or vegetation. The condition of reflection symmetry seldom holds for targets consisting of human-made structures, including urban areas. Hence, for these targets, $\left\langle S_{\mathrm{HH}} S_{\mathrm{HV}}^{*}\right\rangle \neq 0$, and $\left\langle S_{\mathrm{VV}} S_{\mathrm{HV}}^{*}\right\rangle \neq 0$. Yamaguchi et al. [4] introduced the helix scattering model to account for such non-reflection symmetric conditions along with the surface, double-bounce and volume components in their fourcomponent decomposition method.

The volume models considered in the Freeman-Durden [1] and Yamaguchi et al. [4] decomposition techniques are limited to specific types of vegetation due to the assumptions concerning the volume scattering component. Hence, Arii et al. [5] introduced a general canopy model with a generalized probability density function to represent complex canopy structures.

A significant limitation of model-based decompositions is the occurrence of negative power due to improper model fitting. van Zyl et al. [6] proposed a simple modification that ensures that all covariance matrices in the decomposition have non-negative eigenvalues corresponding to physical mechanisms. The non-negative eigenvalue-eigenvector decomposition is used to eliminate additional assumptions that would have been necessary to estimate all the scattering components.

Cui et al. [7] proposed a technique that decomposes the coherency matrix into a volume and two coherent scattering components (characterized by rank-1 matrices). In this technique, determining the volume scattering power leads to the generalized eigendecomposition problem. The non-negative power constraint uniquely defines the minimum eigenvalue as the volume scattering power. 
All model-based decompositions consider prior assumptions about in-scene scatterers. In this respect, the sensitivity of SAR backscatter to target geometry, i.e., target orientation, surface tilt, etc., plays a significant role. These issues are often addressed by compensating target orientation or tilt angle by a linear transformation of the covariance matrix in the decomposition algorithm [8] -[12].

The primary purpose of target orientation compensation is to reduce the effect of the cross-polarized component. In this aspect, Chen et al. [13] proposed general double- and oddbounce scattering models to fit the cross-polarization and offdiagonal terms, separately by their independent orientation angles. Bhattacharya et al. [14] improved the scattering power components of the Singh et al., (G4U) [11] decomposition by utilizing the conventional degree of polarization. In another study, Bhattacharya et al. [15] modified the Yamaguchi et al. [4] scattering power components using a stochastic distance. Ratha et al. [16] proposed a scattering factorization framework for the physical interpretation of target scattering from PolSAR data.

Several additional scattering power decomposition techniques have been proposed with much sophistication to reduce negative power pixels. Such techniques operate by either considering all the elements of the coherency matrix [17] or by introducing compound scattering models involving mixed dipole configuration [18]. Even though these elegant modifications might minimize the occurrence of negative power pixels, they can not eliminate them. Moreover, a significant concern lies in the choice and uniqueness of these models.

Dey et al. [19] first proposed the three-component modelfree scattering power decomposition for full and compact polarimetric SAR data. The authors utilized the $3 \mathrm{D}$ and $2 \mathrm{D}$ Barakat degree of polarization [20], and the elements of the coherency (or covariance) matrix to obtain a target scatteringtype parameter. This parameter was then used to decompose the total scattered power into even-bounce, odd-bounce, and diffused scattering power components. Unlike the volume scattering power component in conventional model-based decompositions, the depolarized part of scattered waves is considered for the diffused scattering component. With this formulation, all the scattering power components are roll-invariant and nonnegative. However, the three-component model-free scattering power decomposition by Dey et al. does not explicitly consider the contribution from asymmetric targets.

Huynen [21] proposed that an average scattered wave from a distributed measurement can be decomposed into an average single-target and a non-symmetric noise component. The helix component exists in this non-symmetric part of the backscattered wave from a distributed target. Later, Yamaguchi et al. [4] introduced the helix as the fourth scattering power component in their decomposition method. A significant proportion of the helix power component is observed over complex urban areas due to the violation of the reflection symmetry condition.

Touzi [22] precisely pointed out the scattering ambiguity between a dihedral and a helix scatterer in a resolution cell using Cloude $\bar{\alpha}$. In this regard, Touzi [22] proposed to assess target asymmetry by using the $\tau_{m}$ parameter. This parameter helps to discriminate a pure dihedral (i.e., $\tau_{m}=0$ ), and helix (i.e., $\tau_{m}= \pm \pi / 4$ ) targets within a resolution cell for which $\bar{\alpha}=\pi / 2$ identically. This study introduces an asymmetric (or helix) scattering-type parameter to resolve the ambiguity concerning the even-bounce and the helix scattering mechanism. We then utilize this parameter to obtain the helix scattering power component disregarded in Dey et al. [19]. Similar to the three-component decomposition method, each power component of this proposed decomposition technique is guaranteed to be non-negative and roll-invariant.

Alongside this, we also propose a new scattering dominancy based clustering algorithm utilizing the odd, even, diffused, and helix scattering power components obtained from the proposed four-component decomposition. We obtain 24 clusters using all possible dominancy permutations of the four scattering power components. This clustering technique assists in understanding the importance of diverse scattering mechanisms based on target characteristics. The technique appropriately captures variations in clusters from one target to another according to their physical and geometrical properties.

This work unfolds as follows. We obtain unique fourcomponent non-model based scattering power decomposition for two data sets in Section III In Section III] we compare the results obtained from the proposed techniques with other existing target characterization parameters and decomposition techniques. Finally, Section IV summarizes the proposed methodologies and concludes by highlighting its advantages and limitations for different SAR data.

\section{Methodology}

We utilize the roll-invariant scattering-type parameter [19] and an asymmetric target characterization parameter along with the elements of the $3 \times 3$ coherency matrix for the derivation of four-component non-model based scattering power components. Subsequently, we use these scattering power components to obtain an unsupervised classification technique. This technique preserves polarimetric scattering dominancy characteristics.

\section{A. Four component scattering power decomposition}

In fully polarimetric (FP) SAR, the $2 \times 2$ complex scattering matrix $\mathbf{S}$ encompasses complete polarimetric information about backscattering from targets for each pixel. It is expressed in the backscatter alignment (BSA) convention in the linear horizontal $(\mathrm{H})$ and linear vertical $(\mathrm{V})$ polarization basis as,

$$
\mathbf{S}=\left[\begin{array}{ll}
S_{\mathrm{HH}} & S_{\mathrm{HV}} \\
S_{\mathrm{VH}} & S_{\mathrm{VV}}
\end{array}\right] \Rightarrow \boldsymbol{k}=V([\mathbf{S}])=\frac{1}{2} \operatorname{Tr}(\mathbf{S} \Psi),
$$

where $k$ is the scattering vector, $V(\cdot)$ is the vectorization operator on the scattering matrix, $\Psi$ is the corresponding basis matrix, and $\operatorname{Tr}$ is the matrix trace (i.e., sum of the diagonal elements of the matrix). Each element of the matrix represents the backscattering response of the target at a specific polarization. The matrix's diagonal elements represent the copolarized scattering information, while the off-diagonal terms represent the cross-polarized information. In the monostatic backscattering case, the reciprocity theorem constrains the scattering matrix to be symmetric, i.e., $S_{\mathrm{HV}}=S_{\mathrm{VH}}$. 
The multi-looked Hermitian positive semi-definite $3 \times 3$ coherency matrix $\mathbf{T}$ is obtained from the averaged outer product of the target vector $\boldsymbol{k}_{P}$ (derived using the Pauli basis matrix, $\Psi_{P}$ ) with its conjugate (i.e., $\mathbf{T}=\left\langle\boldsymbol{k}_{P} \cdot \boldsymbol{k}_{P}^{* T}\right\rangle$ ). Similarly, the $3 \times 3$ covariance matrix $\mathbf{C}$ is obtained from the averaged outer product of the target vector $\boldsymbol{k}_{L}$ (derived using the Lexicographic basis matrix, $\Psi_{L}$ ) with its conjugate (i.e., $\left.\mathbf{C}=\left\langle\boldsymbol{k}_{L} \cdot \boldsymbol{k}_{L}^{* T}\right\rangle\right)$.

$$
\begin{aligned}
& \Psi_{P}=\left\{\sqrt{2}\left[\begin{array}{ll}
1 & 0 \\
0 & 1
\end{array}\right] \quad \sqrt{2}\left[\begin{array}{cc}
1 & 0 \\
0 & -1
\end{array}\right] \quad \sqrt{2}\left[\begin{array}{ll}
0 & 1 \\
1 & 0
\end{array}\right]\right\}, \\
& \Psi_{L}=\left\{2\left[\begin{array}{ll}
1 & 0 \\
0 & 0
\end{array}\right] \quad 2 \sqrt{2}\left[\begin{array}{ll}
0 & 1 \\
0 & 0
\end{array}\right] \quad 2\left[\begin{array}{ll}
0 & 0 \\
0 & 1
\end{array}\right]\right\} .
\end{aligned}
$$

Similarly to the conventional degree of polarization $(0 \leq$ $m \leq 1)$, the 3D Barakat degree of polarization $m_{\mathrm{FP}}$ also characterizes the state of polarization (or purity) of an EM wave. For a completely polarized EM wave $m=1$, and for a completely unpolarized EM wave $m=0$. In between these two extreme cases, the EM wave is partially polarized $(0<m<1)$. The Barakat degree of polarization is linked to the polarimetric contribution of Shannon entropy [23].

Barakat [24] provided an expression of $m$ for the $n \times n$ coherency matrix. This expression is used in this study to obtain the 3D Barakat degree of polarization, $m_{\mathrm{FP}}$ from the $3 \times 3$ coherency matrix $\mathbf{T}$ for FP SAR data:

$$
m_{\mathrm{FP}}=\sqrt{1-\frac{27|\mathbf{T}|}{\operatorname{tr}^{3}(\mathbf{T})}} \in[0,1],
$$

where $|\cdot|$ is the determinant. It should be noted that although this quantity is related to the conventional degree of polarization, it is not the overall degree of polarization for the $n>2$ case as it does not include all the invariants.

In this study, we utilize the $4 \times 4$ real matrix representation to describe backscattering in terms of the Kennaugh matrix $\mathbf{K}$. We can represent the Kennaugh matrix in terms of the elements of the $\mathbf{T}$ matrix as,

$$
\mathbf{K}=\left[\begin{array}{cccc}
\frac{T_{11}+T_{22}+T_{33}}{2\left(T_{12}\right)} & \Re\left(T_{12}\right) & \Re\left(T_{13}\right) & \Im\left(T_{23}\right) \\
\Re\left(T_{13}\right) & \Re\left(T_{23}\right) & \frac{T_{11}-T_{22}+T_{33}}{2} & \Im\left(T_{13}\right) \\
\Im\left(T_{23}\right) & \Im\left(T_{13}\right) & -\Im\left(T_{12}\right) & \frac{-T_{11}+T_{22}+T_{33}}{2}
\end{array}\right],
$$

where $T_{i i}$ for $i=1,2,3$ are the diagonal elements, and $T_{i j}$ for $i \neq j: i, j=1,2,3$ are the off-diagonal elements of $\mathbf{T}$. $\Re$ and $\Im$ denote the real and imaginary parts of a complex number, respectively.

The scattering-type parameter, $\theta_{\mathrm{FP}}$ that is represented using the elements of the $\mathbf{T}$ matrix in [19], can be equivalently expressed using the elements of the $\mathbf{K}$ matrix. For this, let us first consider two free variables, $\eta_{1}$ and $\eta_{2}$ as,

$$
\eta_{1}=\tan ^{-1} \frac{K_{11}-K_{44}}{2 m_{\mathrm{FP}} K_{11}}, \text { and } \eta_{2}=\tan ^{-1} \frac{K_{11}+K_{44}}{2 m_{\mathrm{FP}} K_{11}},
$$

where $K_{11}$ and $K_{44}$ are the elements of $\mathbf{K}$. It may be noted that $\left(K_{11}-K_{44}\right) /\left(2 m_{\mathrm{FP}} K_{11}\right)$ denotes the fraction of power scattered from the regular part 1 of a target with respect to the

\footnotetext{
${ }^{1}$ A general radar target is called regular when the $S_{11}$ and $S_{22}$ elements of the scattering matrix $\mathbf{S}$ are equal in magnitude and phase. In this respect, a sphere is a purely symmetric and regular target, while a corner reflector is non-symmetric and irregular.
}

total polarized power, and that $\left(K_{11}+K_{44}\right) /\left(2 m_{\mathrm{FP}} K_{11}\right)$ denotes the fraction of scattered power from the irregular part of a target with respect to the total polarized power [21]. Hence, by using a simple relationship $\left(\tan \theta_{\mathrm{FP}}=\tan \left(\eta_{1}-\eta_{2}\right)\right)$, we obtain,

$$
\theta_{\mathrm{FP}}=\tan ^{-1} \frac{4 m_{\mathrm{FP}} K_{11} K_{44}}{K_{44}^{2}-\left(1+4 m_{\mathrm{FP}}^{2}\right) K_{11}^{2}} \in\left[-45^{\circ}, 45^{\circ}\right],
$$

where $K_{11}=\left(T_{11}+T_{22}+T_{33}\right) / 2$ and $K_{44}=\left(-T_{11}+\right.$ $\left.T_{22}+T_{33}\right) / 2$. The utilization of the elements of the $\mathbf{K}$ matrix provides equivalency in the formulation of $\theta_{\mathrm{FP}}$ for different SAR data acquisition modes (i.e., full-pol, dual co-pol, and compact-pol). A geometrical interpretation of $\theta_{\mathrm{FP}}$ is given in Appendix A

Apart from the scattering-type parameter $\theta_{\mathrm{FP}}$, we also introduce a scattering asymmetry (helicity) parameter, $\tau_{\mathrm{FP}}$ :

$$
\tau_{\mathrm{FP}}=\tan ^{-1} \frac{\left|K_{14}\right|}{K_{11}} \in\left[0^{\circ}, 45^{\circ}\right]
$$

where $K_{14}=\Im\left(T_{23}\right)$. It can be noted that, according to Huynen [21], the $K_{14}$ element (i.e., the element $F$ in the $\mathbf{T}$ matrix [25]) is the generator of target global twist (helicity). Yamaguchi et al. [4] introduced the $K_{14}$ component in their four-component decomposition theorem for the non-reflection symmetric case that appears over heterogeneous areas. This component is then utilized to characterize the helix power component.

Here, we first derive the helix power component $P_{c}$ (7) by modulating the total polarized power (i.e., $2 m_{\mathrm{FP}} K_{11}$ ) by the scattering asymmetry parameter, $\tau_{\mathrm{FP}}$. The diffused scattering power component $P_{v}$ (8) is obtained as the depolarized fraction (i.e., $1-m_{\mathrm{FP}}$ ) of the total power.

We then obtain the residual power component $P_{r}$ (9), which is equal to the sum of the helix and the diffused power components subtracted from the total scattered power $\left(2 K_{11}\right)$. This residual power component represents the fraction of the polarized scattering power components. This polarized fractional power is then redistributed among odd, $\left(P_{s}\right.$ (10) and even $\left(P_{d}(11)\right)$ power components using the geometrical factor $\left(1 \pm \sin 2 \theta_{\mathrm{FP}}\right)$. This factor depends on the scattering-type parameter $\theta_{\mathrm{FP}}$.

$$
\begin{aligned}
P_{c} & =2 m_{\mathrm{FP}} K_{11} \sin \left(2 \tau_{\mathrm{FP}}\right), \\
P_{v} & =2\left(1-m_{\mathrm{FP}}\right) K_{11}, \\
P_{r} & =2 K_{11}-\left(P_{c}+P_{v}\right) \\
& =2 m_{\mathrm{FP}} K_{11}\left(1-\sin \left(2 \tau_{\mathrm{FP}}\right)\right), \\
P_{s} & =\frac{P_{r}}{2}\left(1+\sin \left(2 \theta_{\mathrm{FP}}\right)\right), \\
P_{d} & =\frac{P_{r}}{2}\left(1-\sin \left(2 \theta_{\mathrm{FP}}\right)\right) .
\end{aligned}
$$

Fig. 1 1 illustrates the procedure as a flowchart.

Let us now characterize $m_{\mathrm{FP}}, \theta_{\mathrm{FP}}$, and $\tau_{\mathrm{FP}}$ along with the four scattering power components for a few particular scattering scenarios:

- For a pure diffused scattering-type, i.e., when $m_{\mathrm{FP}}=0$, then $P_{v}=2 K_{11}=$ Span, and $P_{s}=P_{d}=P_{c}=0$.

- For polarized scattering types, i.e., when $m_{\mathrm{FP}}=1$, two cases arise: 


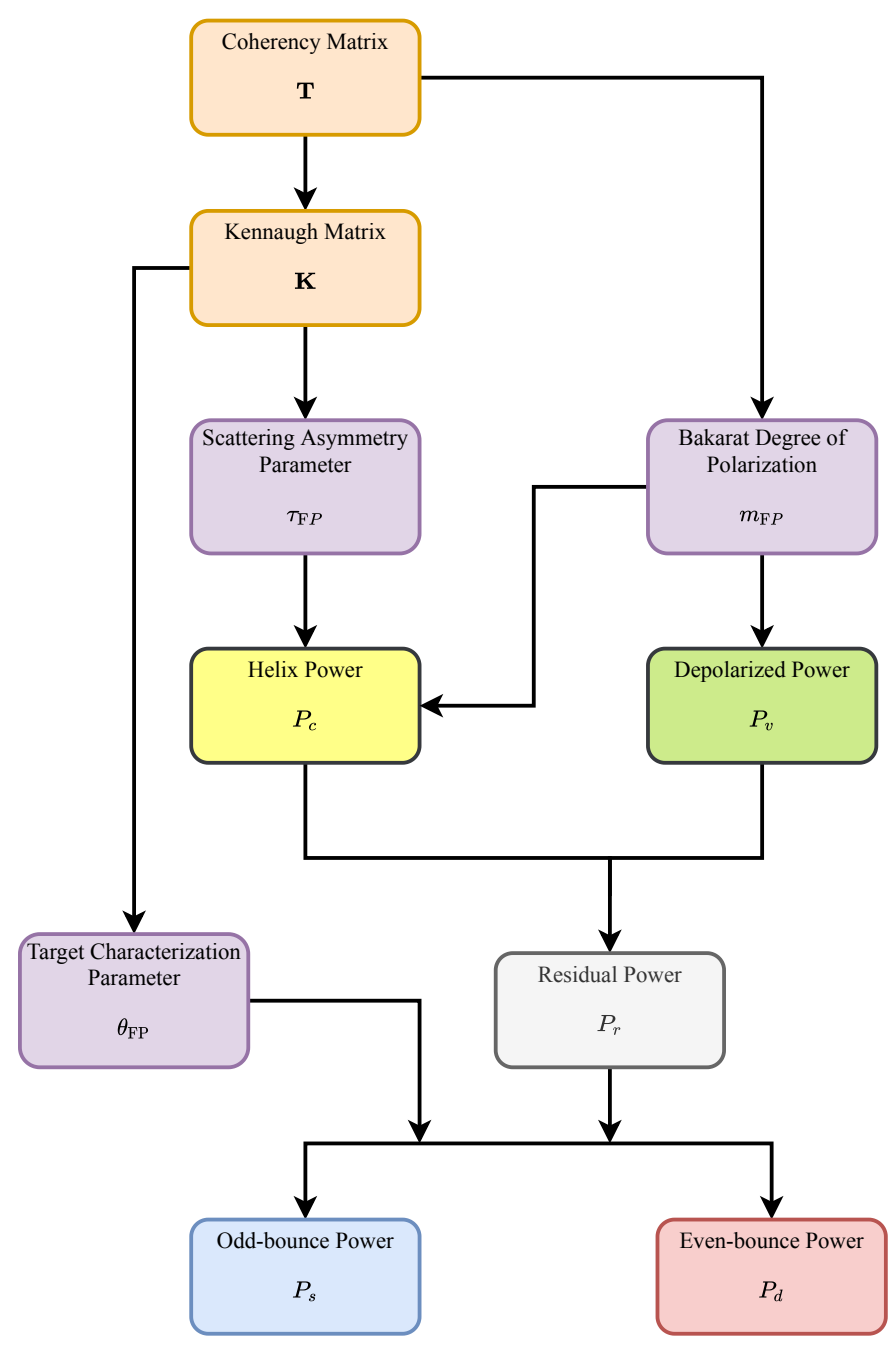

Fig. 1. Flow chart of the model-free four component decomposition technique.

1) if $\theta_{\mathrm{FP}}=45^{\circ}$, and $\tau_{\mathrm{FP}}=0^{\circ}$, then $P_{s}=2 K_{11}=$ Span, and $P_{d}=P_{v}=P_{c}=0$.

2) if $\theta_{\mathrm{FP}}=-45^{\circ}$, and:

a) $\tau_{\mathrm{FP}}=0^{\circ}$, then $P_{d}=2 K_{11}=$ Span, and $P_{s}=$ $P_{v}=P_{c}=0$.

b) $\tau_{\mathrm{FP}}=45^{\circ}$, then $P_{c}=2 K_{11}=$ Span, and $P_{d}=$ $P_{v}=P_{s}=0$. In this case, the scattering is purely asymmetric.

- For $\theta_{\mathrm{FP}}=0^{\circ}$, i.e., when either $m_{\mathrm{FP}}=0$, or $K_{44}=0$, then,

1) if $m_{\mathrm{FP}}=0$, and if $\tau_{\mathrm{FP}}=0^{\circ}$, then $P_{s}=P_{d}=P_{c}=$ 0 , and $P_{v}=2 K_{11}=$ Span

2) if $K_{44}=0$, and if $\tau_{\mathrm{FP}}=0^{\circ}$, then $P_{c}=0$ with $P_{s}=P_{d}$, and $P_{v}$ varies with $m_{\mathrm{FP}} \in[0,1]$.

Fig. 2 shows the variation of the residual power $\left(P_{r}\right)$ with the variation of the scattering asymmetry parameter, $\tau_{\mathrm{FP}}$ for four different values of $m_{\mathrm{FP}}$. For the sake of simplicity, the total received power is fixed at unity (i.e., $2 K_{11}=1$ ). The variation of $P_{r}$ is assessed for $m_{\mathrm{FP}}=1.0,0.7,0.5$ and 0.3 .

It can be seen that as $\tau_{\mathrm{FP}}$ increases, $P_{r}$ decreases for all values of $m_{\mathrm{FP}}$. However, the decreasing slope is steeper for

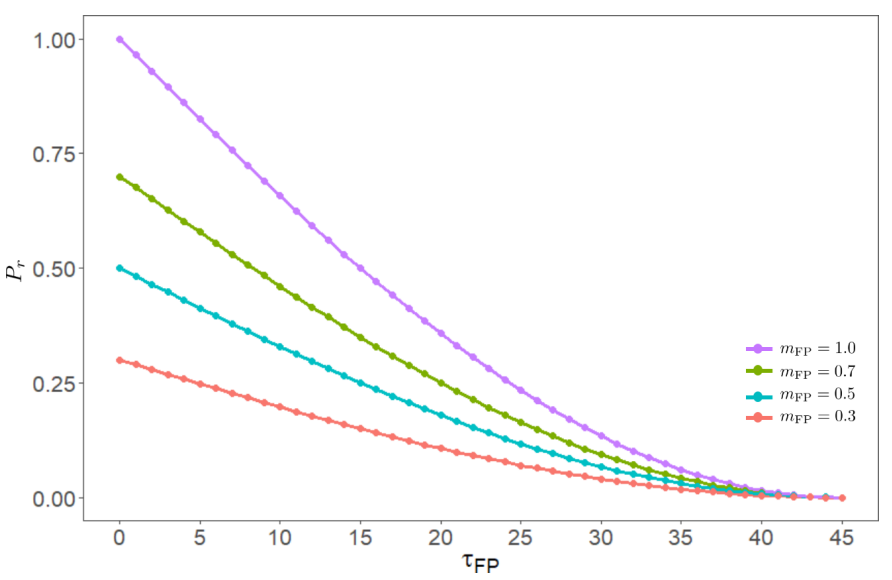

Fig. 2. Variation of residual power, $P_{r}$ with $\tau_{\mathrm{FP}}$ for different values of $m_{\mathrm{FP}}$ with unit Span, i.e., $\left(2 K_{11}=1\right)$.

more coherent targets (i.e., when $m_{\mathrm{FP}}=1.0$ and 0.7 ) than for incoherent targets (i.e., when $m_{\mathrm{FP}}=0.5$ and 0.3 ). This rapid decrease for relatively coherent targets might be due to the steady decline of $m_{\mathrm{FP}}$ with increasing scattering asymmetry. However, regardless the values of $m_{\mathrm{FP}}$, the decrease in $P_{r}$ becomes almost negligible beyond $\tau_{\mathrm{FP}} \approx 35^{\circ}$ and becomes zero at $\tau_{\mathrm{FP}}=45^{\circ}$.

\section{B. Unsupervised Clustering}

In this study, we propose an unsupervised clustering technique based on the permutation of scattering mechanisms according to their dominance. In a previous study, Lee et al. [3] proposed a related unsupervised clustering scheme using the Freeman-Durden scattering power components. However, the clustering technique is entirely based on the information provided by the first dominant scattering mechanism, i.e., surface, double-bounce or volume. Subsequently, pixels in these dominant scattering clusters are further sub-clustered using the Wishart distance. Therefore, a physical justification in the formation of the sub-clusters might not be apparent by using only a statistical measure.

Hence, in this study, the proposed unsupervised clustering scheme provides a natural meaning to each cluster's formation using the information of the dominant scattering mechanism for each pixel. Moreover, successive clusters within each dominant scattering category provide additional physical information for a particular type of landcover. Hence, the unique permutation of the four scattering powers leverages this novel scheme.

First, we divide the pixels into four power categories: 1) dominant even-bounce, 2) dominant odd-bounce, 3) dominant diffused, and 4) dominant helix. Apart from this, we assign a mixed category for pixels whose contribution to the dominant mechanism is ambiguous. We express the ambiguity using a threshold value.

Only pixels within the same scattering category are grouped as a class. This condition warrants the preservation of similar scattering properties. It can be noted that without this restriction, pixels with different scattering characteristics may 
incorrectly get classified into the same class. Fig. 3 describes, in a flowchart, the necessary processing steps of the proposed algorithm. The following subsections provide details about the clustering steps.

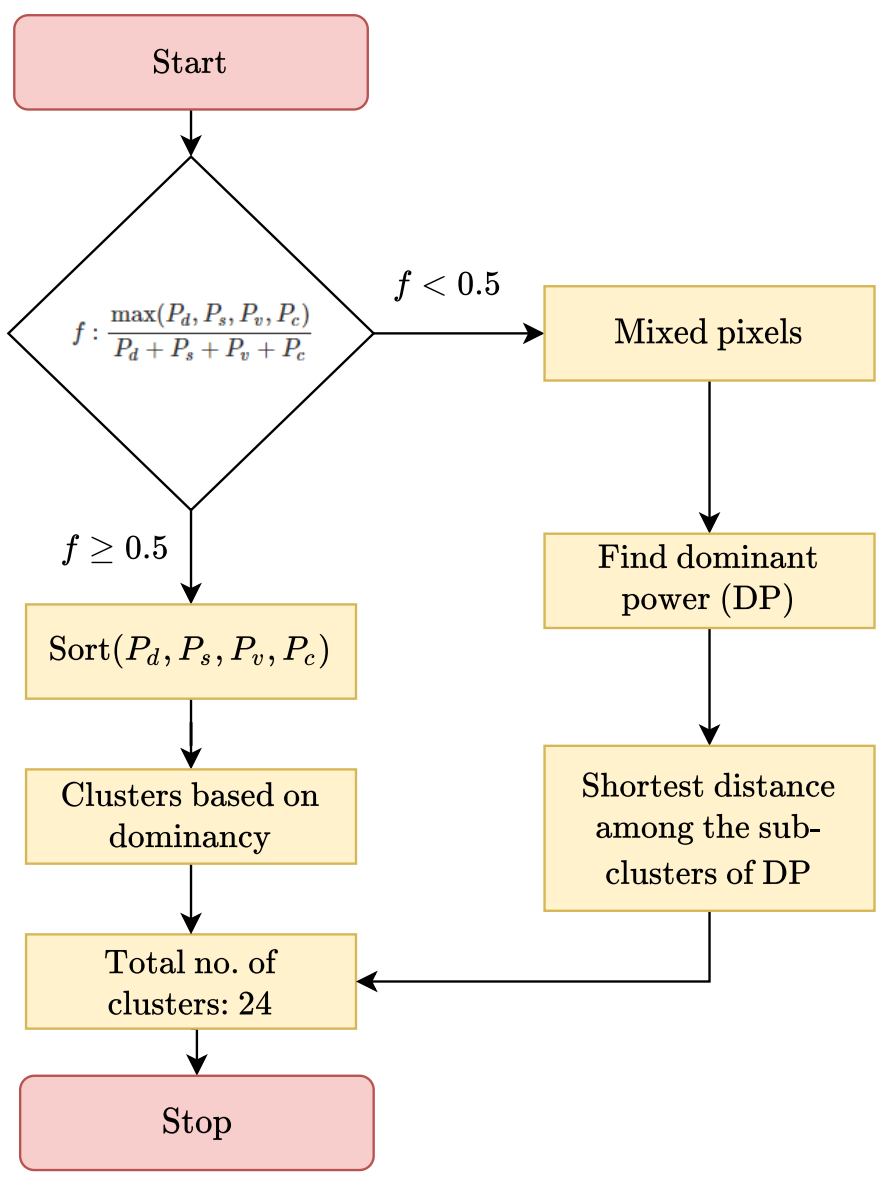

Fig. 3. Flow chart for the proposed clustering framework.

\section{1) Initial clustering:}

- Calculate the normalized scattering power components for each pixel and identify the dominant scattering mechanism.

- A pixel is considered mixed if the dominant scattering mechanism's contribution is less than 0.5.

- Compute the second, third, and fourth dominant scattering powers, and cluster them within the 24 clusters, as shown in Fig. 4

2) Reallocation to clusters of the mixed pixels:

- After forming the initial 24 clusters, calculate the mean values of $P_{d}, P_{s}, P_{v}$, and $P_{c}$ for each cluster.

- Identify the dominant scattering mechanism for the mixed pixels.

- Based on the dominant scattering mechanism, calculate the Euclidean distance among the six sub-clusters of that particular dominant scattering power. Then, assign the pixel to the closest sub-cluster using Euclidean distance. This technique ensures the preservation of the physical scattering phenomenon from a target in a resolution cell.

\section{RESULTS}

In this section, we analyze the scattering power components obtained from the proposed decomposition method using full polarimetric (FP) C-band RADARSAT-2 (RS-2), L-band ALOS PALSAR images over San Francisco (SF), USA, and an X-band TerraSAR-X (TS-X) image over Mumbai, India. Figs. 5. 9, and 10, respectively, show these images.

The C-band RS-2 SF image is acquired with near and far range incidence angles of $28.02^{\circ}$ and $29.81^{\circ}$, respectively. The single look complex (SLC) image is multilooked by a factor of 2 in the range, and 4 in the azimuth to generate an approximately square ground pixel resolution of $20 \mathrm{~m} \times 20 \mathrm{~m}$. The ALOS PALSAR image is multilooked by a unit factor in the range and 7 in the azimuth to generate an approximately square ground pixel resolution of $24 \mathrm{~m} \times 24 \mathrm{~m}$. The TerraSAR-X (TS-X) image is multilooked by a factor of 4 in the range and 4 in the azimuth to generate an approximately square ground pixel resolution of $10 \mathrm{~m} \times 10 \mathrm{~m}$.

\section{A. Variation of $m_{F P}, \tau_{F P}$, and $\theta_{F P}$}

Figure 5 shows the spatial variation of $\theta_{\mathrm{FP}}, \tau_{\mathrm{FP}}$ and $m_{\mathrm{FP}}$ over the C-band SF scene, in which "O" denotes ocean, "U" denotes urban, "OU" denotes oriented urban and "V" denotes vegetation.

We see in Figure 5 that, over the ocean, $\theta_{\mathrm{FP}} \approx 45^{\circ}$, whereas $\tau_{\mathrm{FP}} \approx 0^{\circ}$. These high values of $\theta_{\mathrm{FP}}$ and low values of $\tau_{\mathrm{FP}}$ are due to symmetric coherent type of scattering from the ocean surface which is also evident from the high values of $m_{\mathrm{FP}} \approx 1$. Therefore, we can infer that the scattered wave from region $\mathrm{O}$ is majorly polarized.

Over the urban area, $\mathrm{U}$, the values of $\theta_{\mathrm{FP}}$ are more towards $-45^{\circ}$ and $\tau_{\mathrm{FP}}$ increases marginally. This slight increase in $\tau_{\mathrm{FP}}$ are likely due to asymmetric scatterers present in the urban area. Consequently, a decrease in the value of $m_{\mathrm{FP}}$ is also evident in this area.

However, a significant increase in $\tau_{\mathrm{FP}}$ is evident over the oriented urban (OU) area due to high target asymmetry. Besides, the values of $\theta_{\mathrm{FP}}$ have also decreased compared to the orthogonal urban area. The values of $m_{\mathrm{FP}}$ are also lower than the OU area. In contrast, $\tau_{\mathrm{FP}}$ is lower for the vegetation (V) area than the OU area. Such effect could be due to the symmetric reflection nature of the target for which the spatial variation of $\tau_{\mathrm{FP}}$ is low. The values of $m_{\mathrm{FP}}$ are lower or closer to the OU area due to a certain amount of randomness in scattering from the vegetation area.

Figure 9 shows the spatial variation of $\theta_{\mathrm{FP}}, \tau_{\mathrm{FP}}$ and $m_{\mathrm{FP}}$ over the SF area using ALOS PALSAR data. Over O, the spatial variation of $\theta_{\mathrm{FP}}$ corresponds closely to a coherent target. Also, similarly to RS-2, $\tau_{\mathrm{FP}}$ is very low, and $m_{\mathrm{FP}}$ is very high over O.

A notable change in the variation of $\theta_{\mathrm{FP}}$ is evident over $\mathrm{U}$. A decrease in the values of $m_{\mathrm{FP}}$ is apparent for the ALOS PALSAR data, which is likely due to the long wavelength interaction with urban targets. On the other hand, the values of $\theta_{\mathrm{FP}}$ over OU are similar for both the L-band and C-band data. 


\begin{tabular}{|c|c|c|c|c|c|}
\hline $\begin{array}{c}\mathrm{Z1}: \\
P_{d}>P_{S}>P_{v}>P_{c}\end{array}$ & $\begin{array}{c}\mathrm{Z2}: \\
P_{d}>P_{s}>P_{c}>P_{v}\end{array}$ & $\begin{array}{c}\mathrm{Z3}: \\
P_{d}>P_{v}>P_{s}>P_{c}\end{array}$ & $\begin{array}{c}\mathrm{Z} 4: \\
P_{d}>P_{v}>P_{c}>P_{S}\end{array}$ & $\begin{array}{c}\text { Z5: } \\
P_{d}>P_{c}>P_{S}>P_{v}\end{array}$ & $P_{d}>P_{c}>P_{v}>P_{s}$ \\
\hline $\begin{array}{c}\mathrm{Z7}: \\
P_{s}>P_{d}>P_{v}>P_{c}\end{array}$ & $\begin{array}{c}\text { Z8: } \\
P_{S}>P_{d}>P_{c}>P_{v}\end{array}$ & $\begin{array}{c}\text { Z9: } \\
P_{s}>P_{v}>P_{d}>P_{c}\end{array}$ & $\begin{array}{c}\mathrm{Z} 10: \\
P_{s}>P_{v}>P_{c}>P_{d}\end{array}$ & $\begin{array}{c}\mathrm{Z} 11: \\
P_{s}>P_{c}>P_{d}>P_{v}\end{array}$ & $\begin{array}{c}\mathrm{Z} 12: \\
P_{s}>P_{c}>P_{v}>P_{d}\end{array}$ \\
\hline $\begin{array}{c}\mathrm{Z13}: \\
P_{v}>P_{s}>P_{d}>P_{c} \\
\end{array}$ & $\begin{array}{c}\mathrm{Z} 14: \\
P_{v}>P_{s}>P_{c}>P_{d}\end{array}$ & $\begin{array}{c}\mathrm{Z} 15: \\
P_{v}>P_{d}>P_{s}>P_{c}\end{array}$ & $\begin{array}{c}\mathrm{Z} 16: \\
P_{v}>P_{d}>P_{c}>P_{s}\end{array}$ & $\begin{array}{c}\mathrm{Z} 17: \\
P_{v}>P_{c}>P_{s}>P_{d}\end{array}$ & $\begin{array}{c}\mathrm{Z} 18: \\
P_{v}>P_{c}>P_{d}>P_{s}\end{array}$ \\
\hline $\begin{array}{c}\mathrm{Z19:} \\
P_{c}>P_{d}>P_{S}>P_{v}\end{array}$ & $\begin{array}{c}\mathrm{Z} 20: \\
P_{c}>P_{d}>P_{v}>P_{S}\end{array}$ & $\begin{array}{c}\mathrm{Z} 21: \\
P_{c}>P_{S}>P_{d}>P_{v}\end{array}$ & $\begin{array}{c}\mathrm{Z22:} \\
P_{c}>P_{S}>P_{v}>P_{d}\end{array}$ & $\begin{array}{c}\text { Z23: } \\
P_{c}>P_{v}>P_{d}>P_{s}\end{array}$ & $\begin{array}{c}\mathrm{Z24:} \\
P_{c}>P_{v}>P_{S}>P_{d}\end{array}$ \\
\hline
\end{tabular}

Fig. 4. The proposed 24 cluster sub-divisions by permutation across all scattering power components.

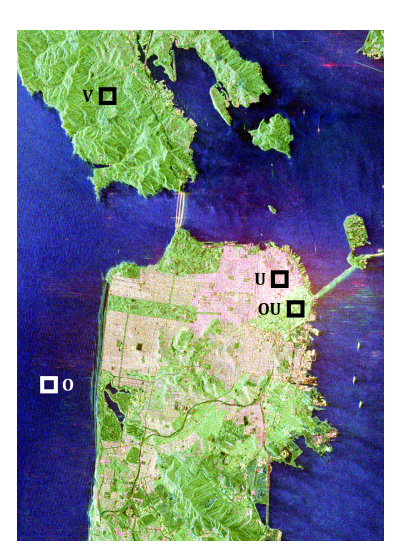

(a) Pauli RGB

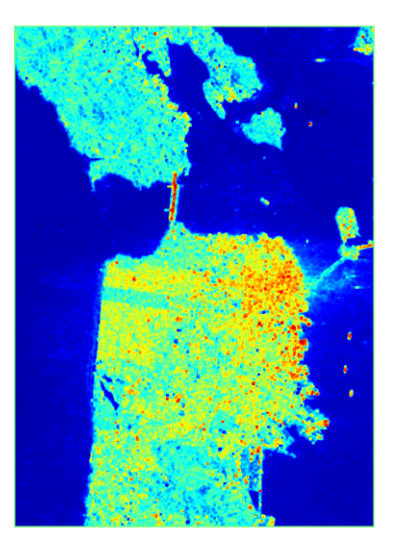

(b) $\theta_{\mathrm{FP}}$

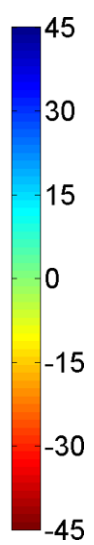

$-45$

Fig. 5. Pauli RGB and different polarimetric descriptor images of RS-2 C-band acquisition over San Francisco, USA.

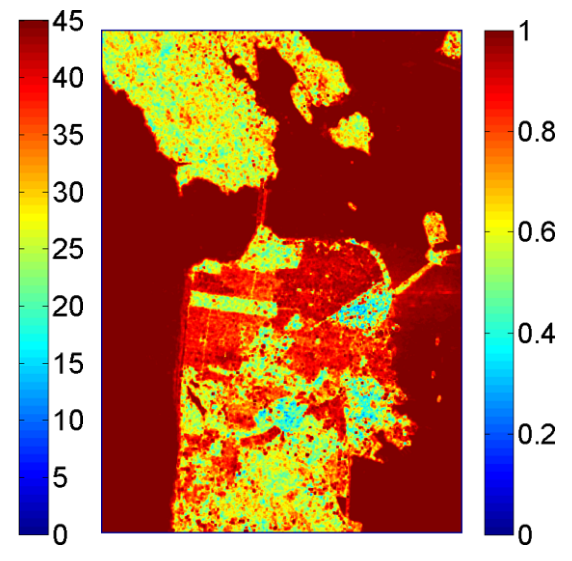

(d) $m_{\mathrm{FP}}$

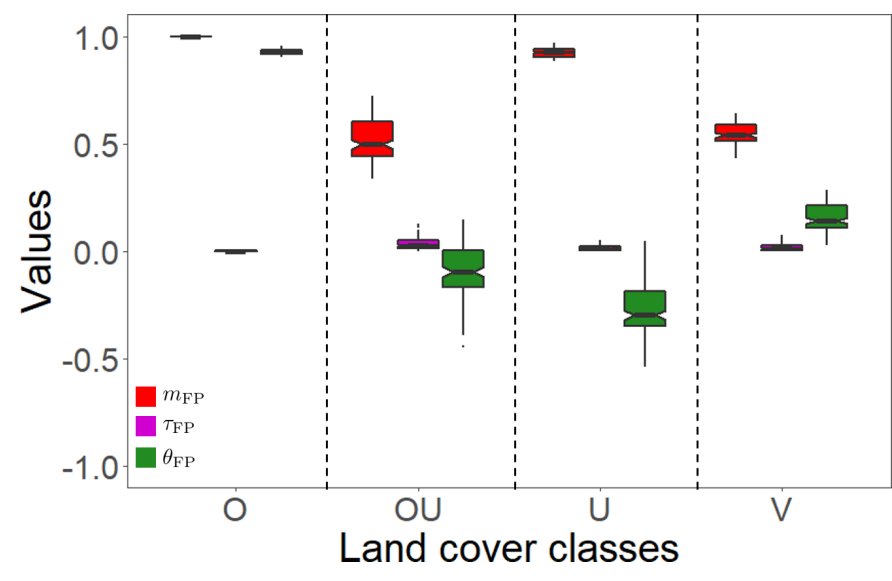

Fig. 6. Box plots of $m_{\mathrm{FP}}, \tau_{\mathrm{FP}}, \theta_{\mathrm{FP}}$ over Ocean (O), Oriented Urban (OU), Urban (U) and Vegetation (V) using RS-2 C-band SAR data. Here, $\tau_{\mathrm{FP}}$ is scaled between 0 to 1 , and, $\theta_{\mathrm{FP}}$ is scaled between -1 to 1 .

Changes over the vegetation area are evident from Figure 9 In comparison to $\theta_{\mathrm{FP}}$ for the C-band RS-2 image, the values of $\theta_{\mathrm{FP}}$ for the L-band PALSAR data reveal multiple scattering from mixed targets. Trees and other vegetation are usually moderately rough on longer wavelengths: the trunk's effect is quite significant while in the shorter wavelengths and, thus, leaves play an important part in the return. This is attributed by the composition of the forest characterized by tree density, and canopy thickness. The scattering properties are also dictated by size, shape and orientation of surface within the forest canopy [26]. A decrease in the value of $m_{\mathrm{FP}}$ is observed compared to the RS-2 data. This suggests a loss in the polarization structure of the EM wave due to complex interaction within the vegetation structure.

TABLE I

MEAN \pm STANDARD DEVIATION OF $\theta_{\mathrm{FP}}, \tau_{\mathrm{FP}}$, AND $m_{\mathrm{FP}}$ OVER OCEAN $(\mathrm{O})$ Urban (U), Oriented URban (OU) and Vegetation (V) USING RS-2

\begin{tabular}{crcc}
\hline & \multicolumn{1}{c}{$\theta_{\mathrm{FP}}$ [degrees] } & $\tau_{\mathrm{FP}}$ [degrees] & $m_{\mathrm{FP}}$ \\
\hline $\mathbf{O}$ & $41.67 \pm 0.48$ & $0.08 \pm 0.04$ & $0.99 \pm 0.01$ \\
$\mathbf{U}$ & $-12.42 \pm 5.34$ & $0.69 \pm 0.43$ & $0.92 \pm 0.02$ \\
$\mathbf{O U}$ & $-4.22 \pm 5.84$ & $1.61 \pm 1.04$ & $0.49 \pm 0.10$ \\
$\mathbf{V}$ & $7.08 \pm 2.67$ & $0.84 \pm 0.73$ & $0.61 \pm 0.03$ \\
\hline
\end{tabular}

For quantitative evaluation, we have randomly sampled 120 pixels from each of the areas (i.e., O, U, OU and V). The box plot in Figure 6 and Figure 7 shows the variations of the three descriptors (i.e, $m_{\mathrm{FP}}, \tau_{\mathrm{FP}}, \theta_{\mathrm{FP}}$ ) over these areas. The mean and standard deviations of the three descriptors for each land cover classes are given in Table 1

Table [1 shows that the mean value of $\theta_{\mathrm{FP}}$ over ocean is $41.67^{\circ}$, which is evident from highly polarized backscatter return $\left(m_{\mathrm{FP}}=0.99\right)$ for the RS-2 data. The standard deviation is also low, which might be due to the ocean surface's 


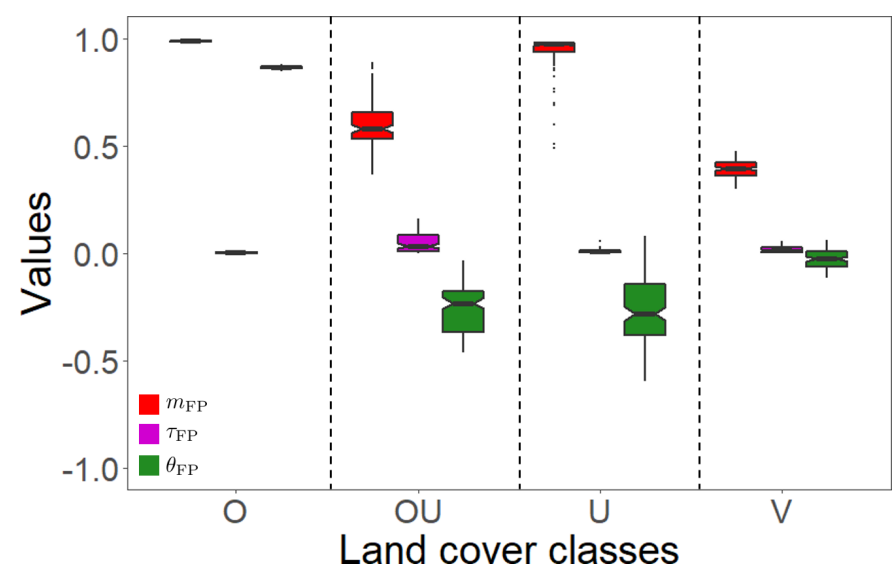

Fig. 7. Box plots of $m_{\mathrm{FP}}, \tau_{\mathrm{FP}}, \theta_{\mathrm{FP}}$ over Ocean (O), Oriented Urban (OU), Urban (U) and Vegetation (V) using ALOS PALSAR L-band SAR data. Here, $\tau_{\mathrm{FP}}$ is scaled between 0 to 1 , and, $\theta_{\mathrm{FP}}$ is scaled between -1 to 1 .

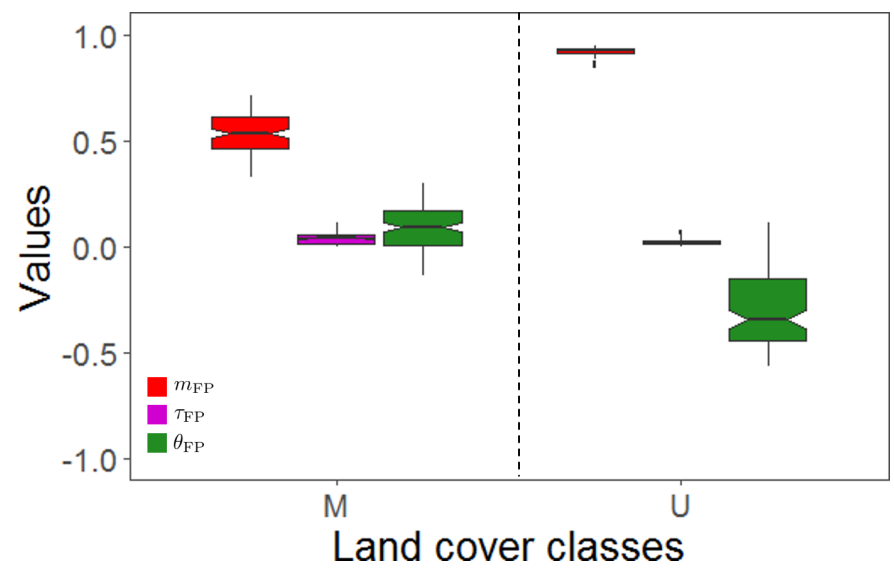

Fig. 8. Box plots of $m_{\mathrm{FP}}, \tau_{\mathrm{FP}}, \theta_{\mathrm{FP}}$ over Mangrove (M) and Urban (U) using TerraSAR-X SAR data. Here, $\tau_{\mathrm{FP}}$ is scaled between 0 to 1 , and, $\theta_{\mathrm{FP}}$ is scaled between -1 to 1 .

homogeneous characteristics. On the other hand, the mean value of $\tau_{\mathrm{FP}}$ is very low (i.e., $0.08^{\circ}$ ), which is due to symmetric scattering from the ocean surface. The non-zero value might be due to the slight roughness generated by the ocean currents.

For the urban area, $m_{\mathrm{FP}}=0.73$ and $\tau_{\mathrm{FP}}=0.69^{\circ}$. However, a good increase in the value of $\tau_{\mathrm{FP}}=1.61^{\circ}$ is observed over OU (Fig. 11). Besides, the standard deviation of $\tau_{\mathrm{FP}}$ is also high due to the non-homogeneous spatial distribution of scatters in this area. Also, the value of $\theta_{\mathrm{FP}}$ has decreased due to the effect of target orientation about the radar line of sight for the $\mathrm{OU}$ area compared to $\mathrm{U}$. The values of $\theta_{\mathrm{FP}}$ for $\mathrm{U}$ is $-12.42^{\circ}$, and for $\mathrm{OU}$ is $-4.22^{\circ}$. Similarly, vegetation being comprised of incoherent scatterers, produces a mean $\theta_{\mathrm{FP}}=7.08^{\circ}$. However, the non-homogeneous nature of vegetation increased the standard deviation to $2.67^{\circ}$. As vegetation produces symmetric scattering, $\tau_{\mathrm{FP}} \approx 0.84^{\circ}$ over this region.

For the L-band ALOS PALSAR data, $\theta_{\mathrm{FP}}=38.93^{\circ}$ over the ocean (O) area, which is lower than the C-band RS-2 data; cf. Table [II. This variation could be due to the difference in the incident wavelength and its interaction with the ocean surface state at the acquisition time. Besides, the value of $\tau_{\mathrm{FP}}$ for the L-band acquisition has also marginally increased $\left(\approx 0.13^{\circ}\right)$ due to the high overall roughness in the ocean. However, the value of $m_{\mathrm{FP}}=0.98$ is similar to that of the C-band data.

TABLE II

MEAN \pm STANDARD DEVIATION OF $\theta_{\mathrm{FP}}, \tau_{\mathrm{FP}}$, AND $m_{\mathrm{FP}}$ OVER OCEAN (O), URBAN (U), ORIENTEd URBAN (OU) AND VEGETATION (V) USING ALOS PALSAR

\begin{tabular}{crcc}
\hline & \multicolumn{1}{c}{$\theta_{\mathrm{FP}}[$ degrees] } & $\tau_{\mathrm{FP}}$ [degrees] & $m_{\mathrm{FP}}$ \\
\hline $\mathbf{O}$ & $38.93 \pm 0.26$ & $0.13 \pm 0.11$ & $0.98 \pm 0.01$ \\
$\mathbf{U}$ & $-14.91 \pm 5.34$ & $0.44 \pm 0.34$ & $0.94 \pm 0.08$ \\
$\mathbf{O U}$ & $-11.49 \pm 7.43$ & $2.29 \pm 2.08$ & $0.60 \pm 0.13$ \\
$\mathbf{V}$ & $-1.09 \pm 2.04$ & $0.76 \pm 0.49$ & $0.39 \pm 0.04$ \\
\hline
\end{tabular}

TABLE III

MEAN \pm STANDARD DEVIATION OF $\theta_{\mathrm{FP}}, \tau_{\mathrm{FP}}$, AND $m_{\mathrm{FP}}$ OVER MANGROVE (M) AND URBAN (U) USING TERRASAR-X

\begin{tabular}{lrcc}
\hline & \multicolumn{1}{c}{$\theta_{\mathrm{FP}}$ [degrees] } & $\tau_{\mathrm{FP}}$ [degrees] & $m_{\mathrm{FP}}$ \\
\hline $\mathbf{M}$ & $4.07 \pm 2.10$ & $0.78 \pm 0.02$ & $0.49 \pm 0.09$ \\
$\mathbf{U}$ & $-13.16 \pm 7.87$ & $0.97 \pm 0.64$ & $0.92 \pm 0.02$ \\
\hline
\end{tabular}

Over $\mathrm{U}$ and $\mathrm{OU}, \theta_{\mathrm{FP}}$ characterizes dihedral scattering mechanism over these areas. Over $\mathrm{U}$, the mean values of $\theta_{\mathrm{FP}}=-14.91^{\circ}$ and over $\mathrm{OU}, \theta_{\mathrm{FP}}=-11.49^{\circ}$. We observe from the standard deviation values, that the uncertainties in $\theta_{\mathrm{FP}}$ over these areas are higher than that of $\mathrm{O}$ and $\mathrm{V}$. This might be due to the inherent scattering asymmetry from these targets. High values of $\tau_{\mathrm{FP}}$ also confirm this asymmetric scattering nature from these targets (Fig. 11). It can be seen from Table II that the mean value of $\tau_{\mathrm{FP}}=0.44^{\circ}$ for the urban area, whereas $\tau_{\mathrm{FP}}=2.29^{\circ}$ over the oriented urban area.

Table II shows that over $\mathrm{V}, \theta_{\mathrm{FP}}=-1.09^{\circ}$ characterizes scattering from even multiple bounces. This could be due to the penetration capability of the L-band wave inside the vegetation canopy. Besides, the mean value of $\tau_{\mathrm{FP}}$ has decreased over this area compared to $\mathrm{U}$ and $\mathrm{OU}$ due to scattering symmetry property of the vegetation surface.

Similarly, the variation of $\theta_{\mathrm{FP}}, \tau_{\mathrm{FP}}$ and $m_{\mathrm{FP}}$ are analyzed over the mangrove ('M') and urban ('U') areas using the TS-X data is shown in Table III Over M, low values of $\theta_{\mathrm{FP}}=4.07^{\circ}$ and $m_{\mathrm{FP}}=0.49$ typically represent the amount of depolarization due to scattering from randomly oriented branches of the canopies. Also, the reflection symmetric property of the mangrove canopy surface is apparent from the low value of $\tau_{\mathrm{FP}}$ as $0.78^{\circ}$.

On the other hand, the degree of polarization over $\mathrm{U}$ is 0.92 . This is likely due to the coherent nature of the scattering from buildings. An even-bounce scattering mechanism is also evident with $\theta_{\mathrm{FP}}=-13.16^{\circ}$. A slight increase in reflection asymmetry is visible with a marginal increase in the values of $\tau_{\mathrm{FP}}$ compared to $\mathrm{M}$.

\section{B. Variation of scattering power components}

Fig. 12 shows the scattering power components obtained from the proposed model-free four-component decomposition 


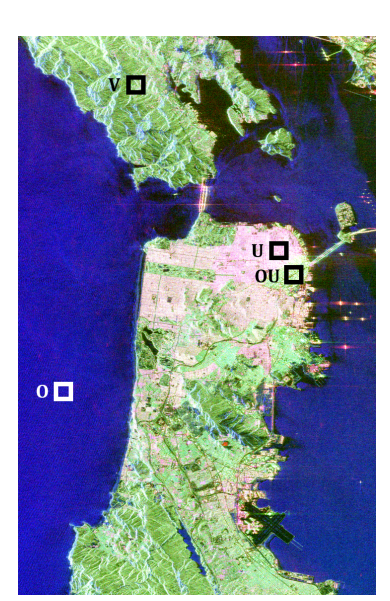

(a) Pauli RGB

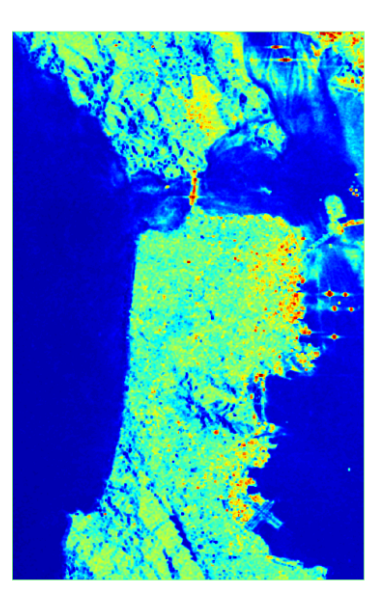

(b) $\theta_{\mathrm{FP}}$

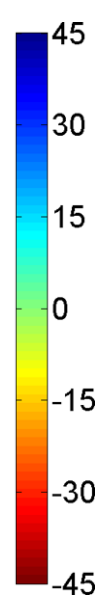

45

Fig. 9. Pauli RGB and different polarimetric descriptor images of ALOS PALSAR L-band acquisition over San Francisco, USA.

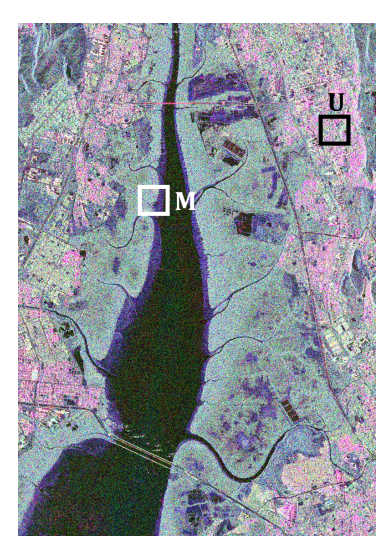

(a) Pauli RGB

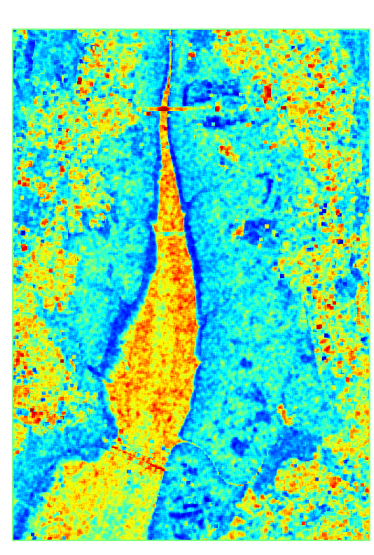

(b) $\theta_{\mathrm{FP}}$

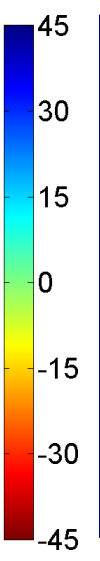

$-45$

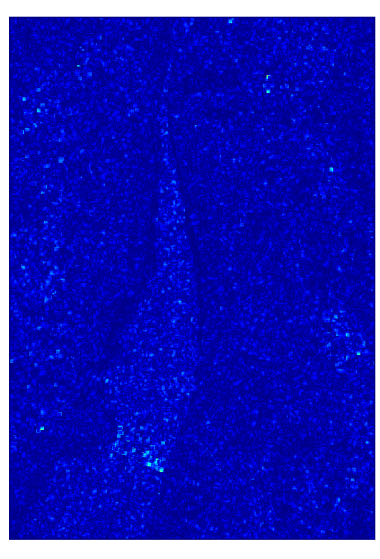

(c) $\tau_{\mathrm{FP}}$
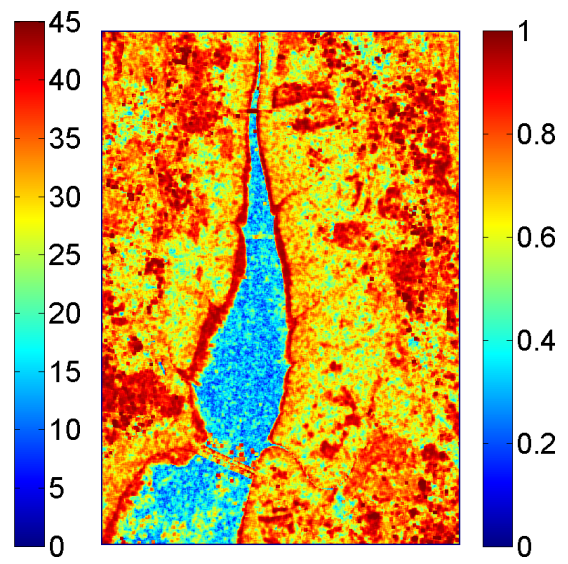

(d) $m_{\mathrm{FP}}$

Fig. 10. Pauli RGB and different polarimetric descriptor images of TerraSAR-X acquisition over Mumbai, India.

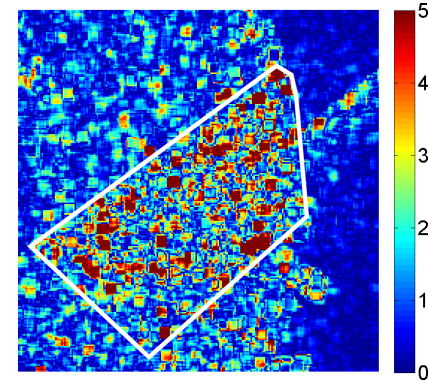

(a) RS-2

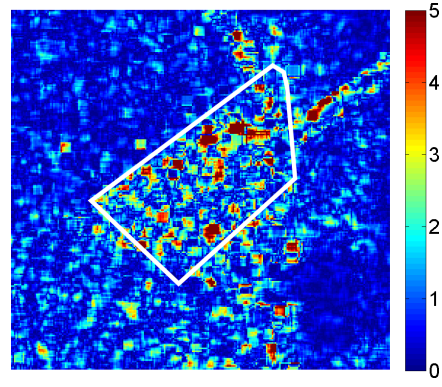

(b) ALOS PALSAR
Fig. 11. Variation of $\tau_{\mathrm{FP}}$ over OU for RS-2 and ALOS PALSAR data. The zoomed region is shown with a white boundary.

(MF4CF) over $\mathrm{O}, \mathrm{U}, \mathrm{OU}$ and $\mathrm{V}$ areas using the $\mathrm{RS}-2$ data. Here, we have compared the power components of $\mathrm{MF} 4 \mathrm{CF}$ with the Yamaguchi four-component decomposition technique with rotation of the coherency matrix [10] (Y4R), the General four-component scattering power decomposition (G4U) [27], the Adaptive general four-component scattering power decomposition (AG4U) [14], and the Six-component scattering power decomposition (i6SD) [17] techniques.

For RS-2 data over the ocean surface, O, the odd-bounce scattering power $P_{s}$, is $83.3 \%$ for $\mathrm{Y} 4 \mathrm{R}, 84.3 \%$ for $\mathrm{G} 4 \mathrm{U}$, $95.22 \%$ for $\mathrm{AG} 4 \mathrm{U}$ and $87.63 \%$ for $\mathrm{i6SD}$ whereas it is $99.39 \%$ for MF4CF. Therefore, the odd-bounce scattering power has increased by $\approx 12 \%$ for MF4CF compared to other decomposition techniques.

For Y4R and G4U, over the ocean surface, the amount of helix scattering powers are $5.1 \%$ and $5.3 \%$ respectively. The asymmetric power component is $0.01 \%$ for MF4CF, which is similar to the helix scattering power component for AG4U, and i6SD. Therefore, MF4CF, AG4U and i6SD can equivalently indicate scattering characteristics of the ocean surface correctly. This observation is justified by the fact that the scattering from the ocean surface is symmetric odd-bounce.

On the other hand, over the urban area, U and OU, the even-bounce scattering power and the asymmetric scattering power components have also increased for MF4CF. However, significant differences among the scattering powers can be observed over OU. Over OU, Y4R, G4U, AG4U and i6SD show dominant volume scattering component $(\approx 90.8 \%$, 


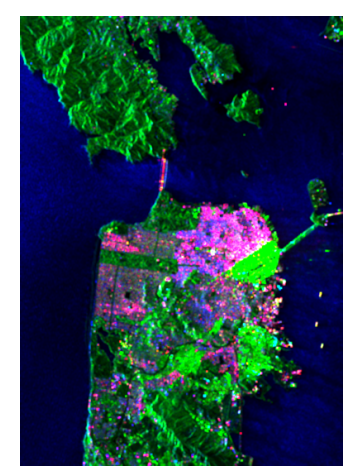

(a) $\mathrm{Y} 4 \mathrm{R}$

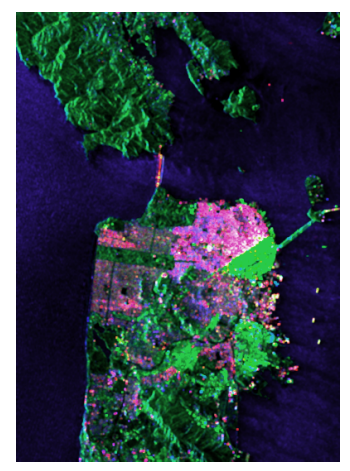

(b) G4U

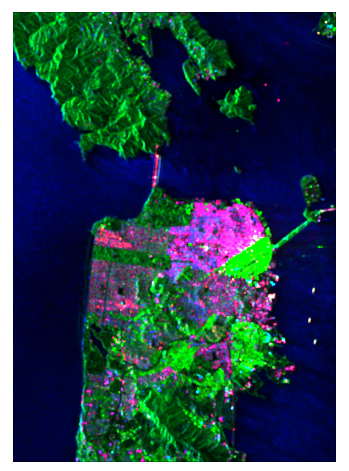

(c) AG4U

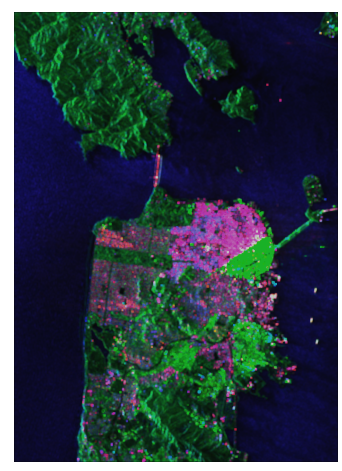

(d) i6SD

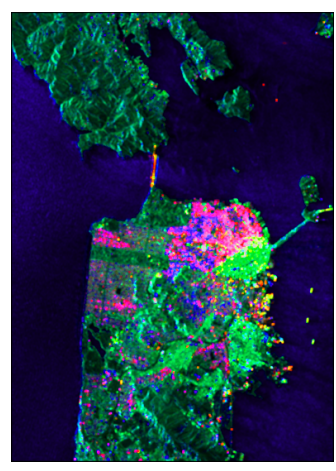

(e) MF4CF

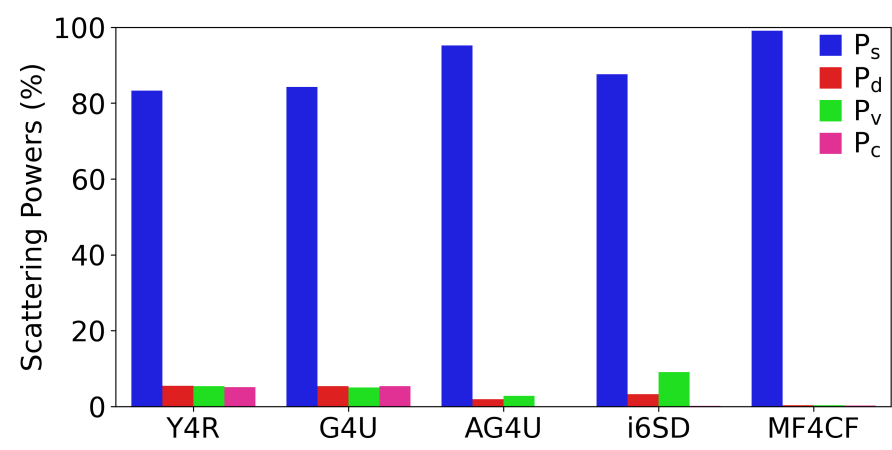

(f) $\mathrm{O}$

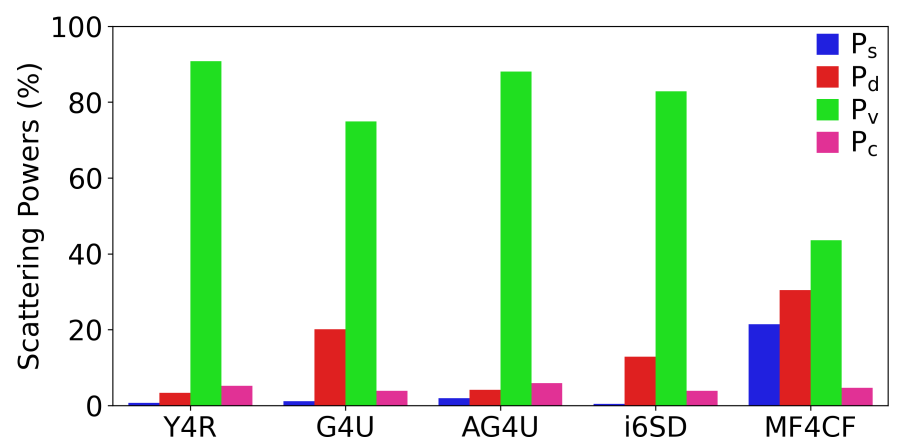

(h) $\mathrm{OU}$

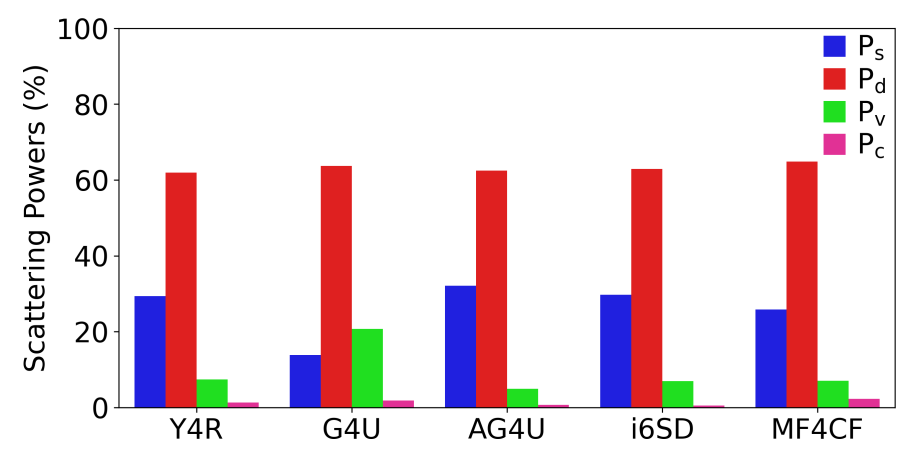

(g) $U$

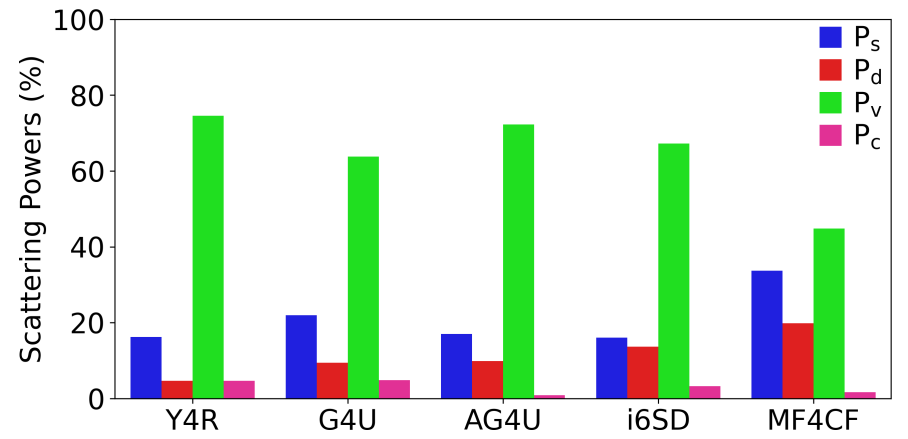

(i) $\mathrm{V}$

Fig. 12. Decomposed scattering power components over Ocean (O), Urban (U), Oriented Urban (OU) and Vegetation (V) using Y4R, G4U, AG4U, i6SD and MF4CF decomposition techniques using RS-2 data.

$\approx 74.89 \%, \approx 88.08 \%$ and $\approx 82.89 \%$, respectively), while MF4CF shows $P_{v}=43.60 \%$. In comparison to other decompositions, the even-bounce scattering power from MF4CF has also increased by $\approx 15 \%$, while there is an increase of $\approx 26 \%$ compared to AG4U.

It is noteworthy to observe that although Y4R, G4U, AG4U and i6SD compensate for the orientation of the dihedral targets, the volume scattering power components from these two techniques are nearly twice the diffused scattering power component of MF4CF. Such an effect might be due to the utilization of the degree of polarization in the formulation of the roll-invariant scattering power components. Moreover, oriented urban areas display a high amount of scattering asymmetry [28]. This fact is evident from the asymmetric scattering power component $\left(P_{c}\right)$ from MF4CF.

High even-bounce scattering power over V $(19.81 \%)$ is due to the interaction of the electromagnetic wave with ground and vegetation trunk as well as ground and vegetation branches. The increase in the odd-bounce scattering power is due to the polarized scattered wave from leaves and foliage. Moreover, the overall diffused scattering power component has decreased in comparison to other decomposition technique. Therefore, the degree of depolarization can adequately provide diverse scattering characteristics than the cross-pol component (i.e., HV) alone that is utilized in the volume scattering model.

Alongside this, as stated earlier, that vegetation is considered to be reflection-symmetric. Therefore, the contribution of the asymmetric scattering power component is only $1.64 \%$. In contrast, Y4R, G4U and i6SD show a helix scattering power component of $\approx 4 \%$. MF4CF and AG4U characterize vegetation surface as more symmetric scatterer than Y4R. Moreover, MF4CF better captures the polarized scattered wave from the vegetation canopy, omitted by Y4R and AG4U.

Figure 13 shows the decomposed power components over 


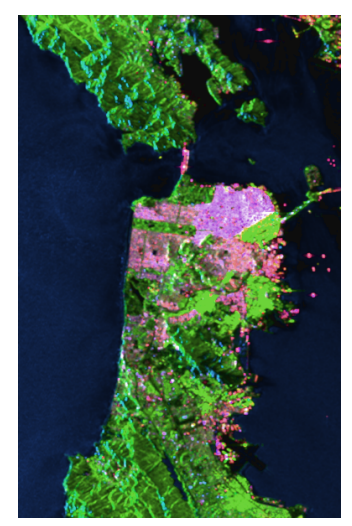

(a) $\mathrm{Y} 4 \mathrm{R}$

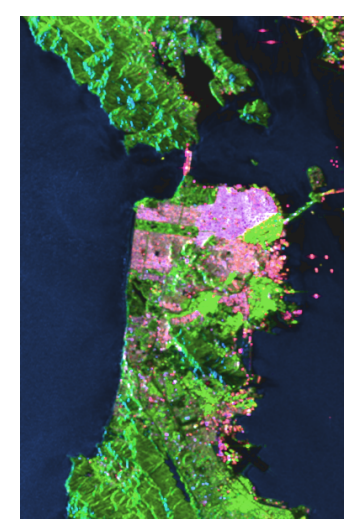

(b) G4U

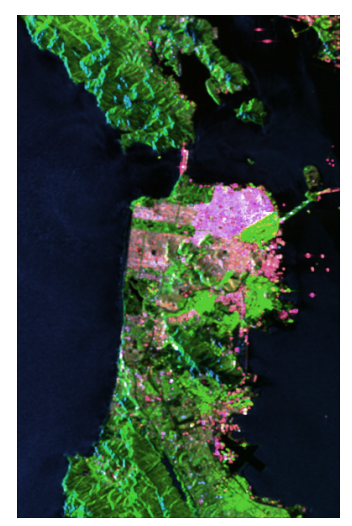

(c) AG4U

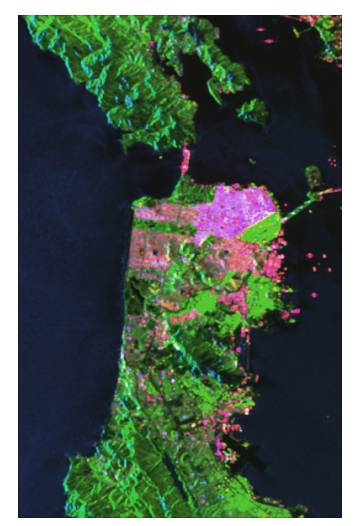

(d) i6SD

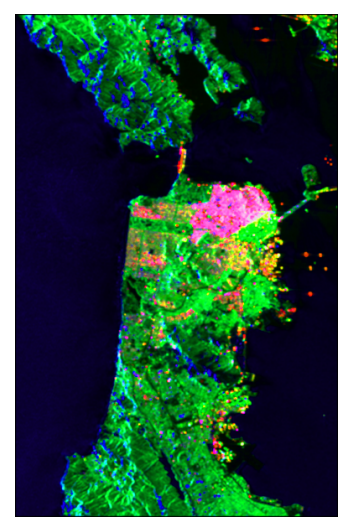

(e) MF4CF

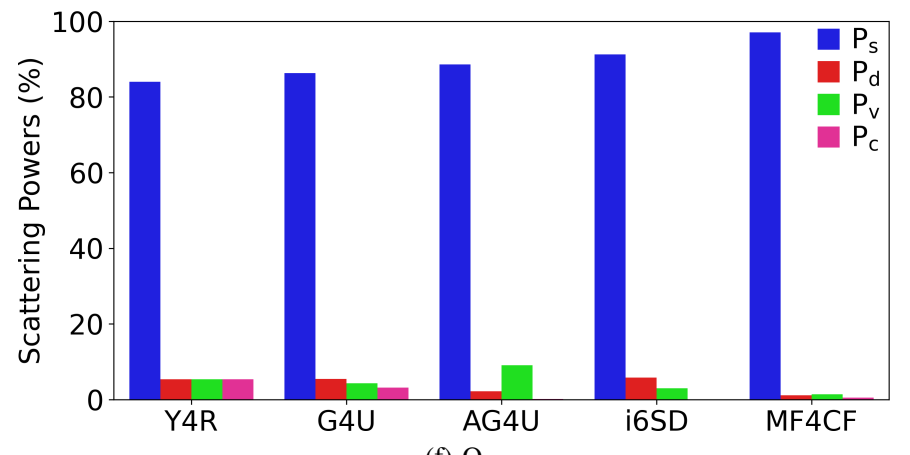

(f) $\mathrm{O}$

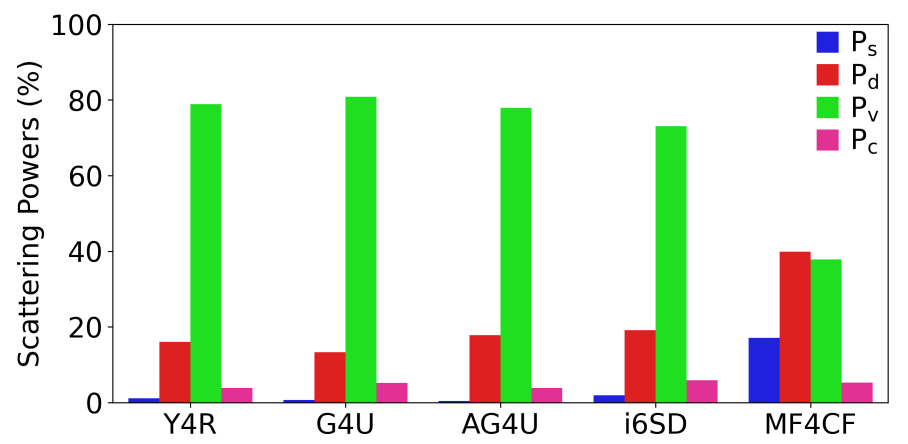

(h) $\mathrm{OU}$

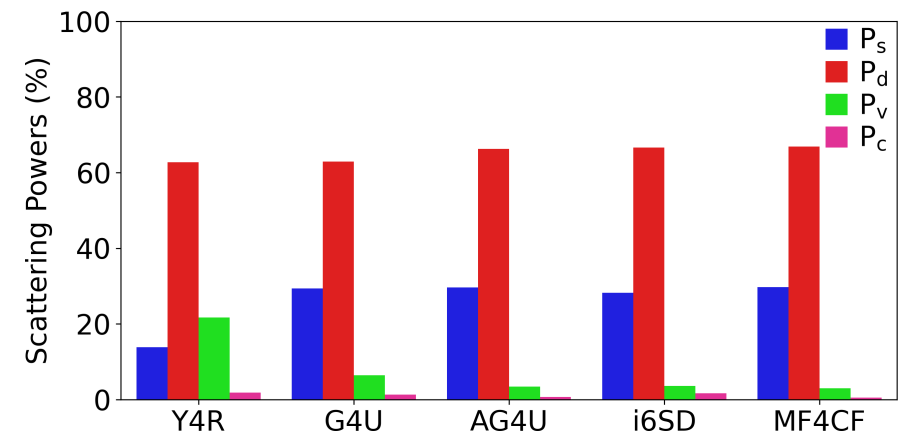

(g) $U$

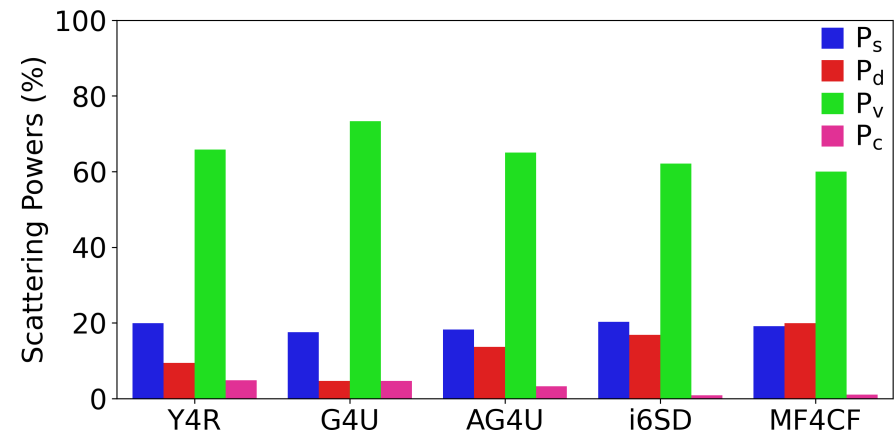

(i) $\mathrm{V}$

Fig. 13. Decomposed scattering power components over Ocean (O), Urban (U), Oriented Urban (OU) and Vegetation (V) using Y4R, G4U, AG4U, i6SD and MF4CF decomposition techniques using ALOS PALSAR data.

$\mathrm{O}, \mathrm{U}, \mathrm{OU}$ and $\mathrm{V}$ using ALOS PALSAR data. The dominant odd-bounce scattering power is evident over O. However, $P_{s}$ has decreased by $\approx 2 \%$ as compared to the RS-2 data. This decrease in the $P_{s}$ value might be because the ocean surface roughness is more apparent in longer wavelengths. Consequently, $P_{c}$ has also marginally increased by $0.03 \%$ compared to the RS-2 data. On the other hand, Y4R and AG4U show significant volume $(5.3 \%$ and $9.04 \%)$ scattering power, which might be due to the assumption of a specific volume scattering model that might not be adequate for this scenario.

Over $\mathrm{U}$, the sample mean of $P_{d}$ obtained from MF4CF has increased by $4 \%$ as compared to $\mathrm{Y} 4 \mathrm{R}$ and $\mathrm{G} 4 \mathrm{U}$, and by $\approx 0.2 \%$ as compared to AG4U and i6SD. Hence, the values of $P_{d}$ are quite similar for both MF4CF, AG4U and i6SD over U. We may justify such an outcome because, in MF4CF, we explicitly use the degree of polarization to calculate the scattering power components. It may be noted that, in AG4U, we use the degree of polarization only as a criterion to compute the power component, whereas its usefulness is absent in any other decompositions Therefore, the Barakat degree of polarization provides essential information in calculating the scattering power components by utilizing the polarization structure's knowledge in the scattered wave from urban areas.

The diffused scattering power has decreased over this area compared to the volume scattering power component of Y4R and G4U. Nevertheless, the volume scattering component of AG4U, i6SD and the diffused component of MF4CF are similar. In contrast, the asymmetric power component, $P_{c}$, is similar to the helix scattering power component of Y4R and G4U. The percentages of $P_{c}$ and the helix scattering power components are $1.60 \%, 1.8 \%$ and $1.3 \%$, respectively. 


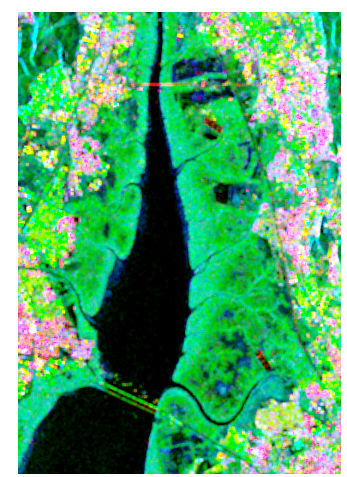

(a) Y4R

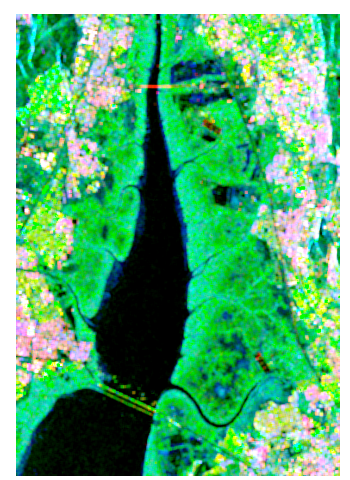

(b) G4U

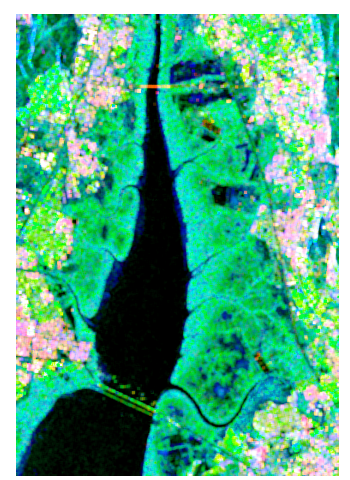

(c) AG4U

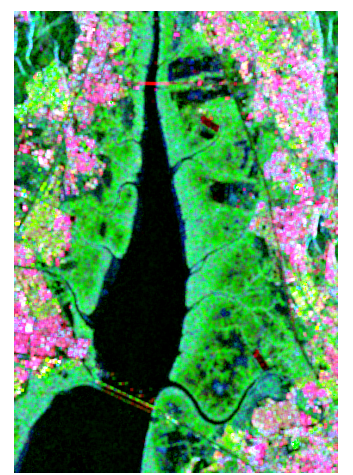

(d) i6SD

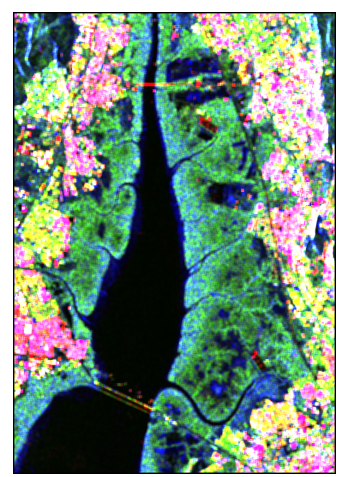

(e) MF4CF

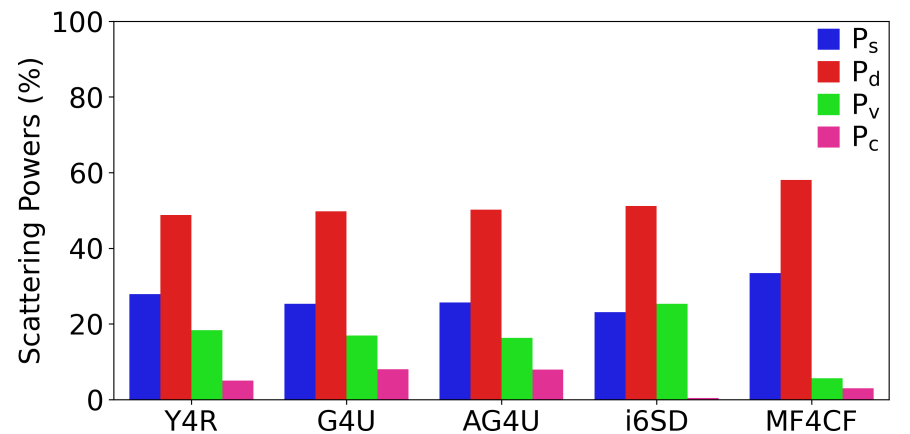

(f) $\mathrm{U}$

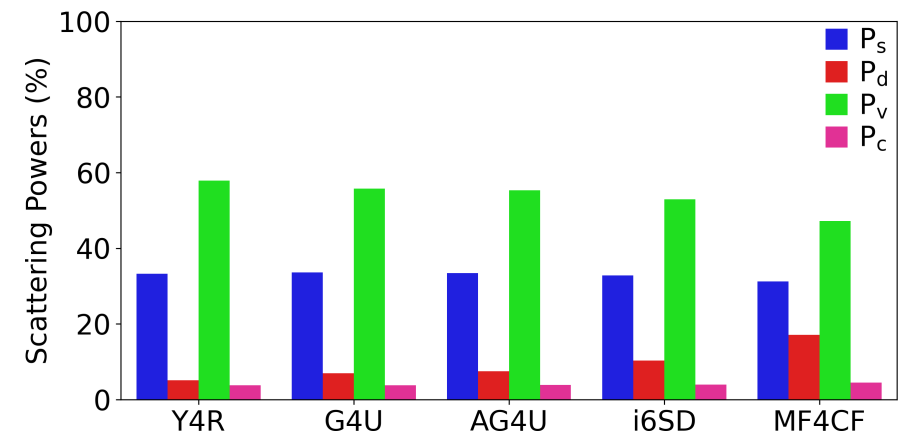

(g) $\mathrm{M}$

Fig. 14. Decomposed scattering power components over Urban (U) and Mangrove (M) using Y4R, G4U, AG4U, i6SD and MF4CF decomposition techniques using TerraSAR-X data.

Fig. 13 shows significant differences between the evenbounce scattering power of MF4CF and the double-bounce scattering power of Y4R, G4U, AG4U and i6SD over OU. Besides, the difference between the diffused and the volume scattering powers from the L-band $(\approx 40 \%)$ is lower than that of the C-band. It can be noted that $P_{d}$ obtained from MF4CF is still the dominant scattering power component $(39.90 \%)$ over OU. It is noteworthy to observe that the scattering asymmetry due to the urban area's orientation about the radar line of sight is more prominent in MF4CF than other decomposition techniques. The percentage of $P_{c}$ and helix scattering components are $5.22 \% 3.88 \%, 5.2 \%, 3.87 \%$ and $5.9 \%$, respectively for MF4CF, Y4R, G4U, AG4U and i6SD.

Similarly, over vegetation (V), a marginal difference is evident in the diffused scattering power component of MF4CF $(59.96 \%)$ and the volume scattering component of Y4R (65.8\%). The difference between the volume and the diffused power components from $\mathrm{AG} 4 \mathrm{U}$ to $\mathrm{MF} 4 \mathrm{CF}$ is $\approx 6 \%$. However, $P_{d}$ has increased by $\approx 10 \%$ from $\mathrm{Y} 4 \mathrm{R}, \mathrm{G} 4 \mathrm{U}$ to $\mathrm{MF} 4 \mathrm{CF}$, $\approx 6 \%$ from $\mathrm{AG} 4 \mathrm{U}$ to $\mathrm{MF} 4 \mathrm{CF}$, and $\approx 3 \%$ from $\mathrm{i} 6 \mathrm{SD}$ to MF4CF.

As stated earlier, such a high value of $P_{d}$ is due to the forest's ground canopy interactions. The explicit utilization of the degree of polarization in the formulation of MF4CF might have adequately accounted for the polarization state information in the scattered EM wave. On the other hand, similar to the C-band data, the mean value of $P_{c}$ is $1.03 \%$, whereas the helix power components of $\mathrm{Y} 4 \mathrm{R}$ is $3.2 \%$ and for AG4U is $4.8 \%$. However, the helix power is similar for both
i6SD and MF4CF. Therefore, $P_{c}$ characterizes vegetation as a more symmetric scatterer than Y4R and AG4U. However, the disparity between the diffused scattering power component of MF4CF and the volume scattering power components of other decompositions is marginal. This variation might be due to the L-band wave's scattering properties inside the vegetation canopy that is equivalently characterized by the volume scattering model and the degree of polarization measure.

Similarly, the scattering power components over Mumbai, India, using TerraSAR-X data is compared in Fig. 14. Here we have shown the decomposition results over urban (U) and mangrove (M) areas. Like L-band and C-band data, the $P_{d}$ component using MF4CF over $\mathrm{U}$ for $\mathrm{X}$-band data has increased compared to Y4R, G4U, AG4U, and i6SD. The differences between the $P_{d}$ of MF4CF and other decomposition techniques are $\approx 7 \%$. On the other hand, the $P_{v}$ components have also decreased by $\approx 10 \%$ for MF4CF compared to Y4R, G4U, AG4U and i6SD.

In contrast, the trend of scattering power components over $\mathrm{M}$ is similar for different decomposition methods. However, the $P_{v}$ component has decreased by $\approx 14 \%$ compared to other decomposition methods. This decrease in the $P_{v}$ component might be due to the significant polarization structure in the returned electromagnetic wave. As a result, the polarized even and odd bounce components have increased over M compared to Y4R, G4U, AG4U and i6SD. The increase in the $P_{d}$ and $P_{s}$ components are nearly $3 \%$ and $12 \%$, respectively. This $P_{d}$ component is due to the electromagnetic wave interaction with the underneath water and branches. The $P_{s}$ component 
is due to the interaction of the electromagnetic wave with the leaves at the top of the canopy layers.

Hence, the proposed method suitably characterizes different scattering properties from targets compared to Y4R, G4U, AG4U and i6SD. Also, the $P_{c}$ power component can accurately represent scattering asymmetry over the desired targets. It is noteworthy that the inclusion of the scattered wave's polarization state has improved the target characterization ability of MF4CF compared to other techniques.

\section{Clustering}

Here we discuss a clustering scheme utilizing the four scattering power components, $P_{d}, P_{s}, P_{v}$, and $P_{c}$ obtained from the $\mathrm{C}-$, L-, and X-band PolSAR datasets. To assess and validate the clustering results, we utilize different combinations of entropy $(H)$ and anisotropy $(A)$, and the first dominant scattering-type parameter $\left(\alpha_{s}^{1}\right)[25]$. We used the following combinations of $H$ and $A$ :

- The presence of a single dominant scattering process is described by $(1-H)(1-A)$.

- A random scattering process is modeled by $H(1-A)$.

- The presence of two scattering mechanisms with the same probability relates to $H A$.

- Two scattering mechanisms with a dominant process is described by $(1-H) A$.

Fig. 15 shows the unsupervised map using RS-2 and ALOS PALSAR data over SF, and TerraSAR-X data over Mumbai, India.

TABLE IV

PERCENTAGE OF PIXELS IN DIFFERENT CLUSTERS OVER OCEAN SURFACE USING RS-2 AND ALOS PALSAR DATA (Z7: $P_{s}>P_{d}>P_{v}>P_{c} ; \mathrm{Z} 9$ : $\left.P_{s}>P_{v}>P_{d}>P_{c}\right)$

\begin{tabular}{|c|c|c|c|}
\hline \multicolumn{2}{|r|}{ RS-2 } & \multicolumn{2}{|c|}{ ALOS PALSAR } \\
\hline Class & Percent (\%) & Class & Percent (\%) \\
\hline $\mathrm{Z7}$ & 95.40 & Z7 & 91.60 \\
\hline Z9 & 4.60 & Z9 & 8.40 \\
\hline
\end{tabular}

Table IV shows that the two different clusters, Z7 and Z9, are common for both RS-2 and ALOS PALSAR datasets. In both of them, the odd-bounce scattering power component is the first dominant. However, the second dominant components are the even-bounce scattering for $\mathrm{Z} 7$ and the diffused scattering for Z9. The combination $(1-H)(1-A)$ is higher than any other for both datasets, suggesting a single dominant scattering process. Moreover, we observe $\alpha_{s}^{1}=3.29^{\circ}$ and $\alpha_{s}^{1}=4.16^{\circ}$ for RS-2 and PALSAR data, respectively. However, the value of $(1-H) A$ is apparent, which indicates that there might exist two scattering mechanisms with a dominant process.

In the context of the ocean surface, the existence of other scattering power components might be due to surface roughness. The ocean current's ridge produces a marginal amount of even-multiple scattering that might cause the even-bounce scattering power as the second dominant. On the other hand, complex ocean surface roughness near the shoreline favours diffused scattering power components as the second dominant in a few pixels. However, being a reflection-symmetric surface, the $P_{c}$ power is always the fourth dominant scattering component over the ocean surface.

TABLE V

PERCENTAGE OF PIXELS IN DIFFERENT CLUSTERS OVER URBAN USING RS-2 AND ALOS PALSAR DATA (Z1: $P_{d}>P_{s}>P_{v}>P_{c}$; Z2: $\left.P_{d}>P_{s}>P_{c}>P_{v} ; \mathrm{Z7}: P_{s}>P_{d}>P_{v}>P_{c}\right)$

\begin{tabular}{|c|c|c|c|}
\hline \multicolumn{2}{|r|}{ RS-2 } & \multicolumn{2}{|c|}{ ALOS PALSAR } \\
\hline Class & Percent (\%) & Class & Percent (\%) \\
\hline $\mathrm{Z} 1$ & 49.70 & Z1 & 76.94 \\
\hline $\mathrm{Z} 2$ & 16.90 & $\mathrm{Z} 2$ & 23.06 \\
\hline $\mathrm{Z7}$ & 33.40 & $\mathrm{Z7}$ & 0.00 \\
\hline
\end{tabular}

Table $\mathrm{V}$ shows that over the urban area, pixels are clustered in $\mathrm{Z} 1, \mathrm{Z} 2$ and $\mathrm{Z7}$ for the RS-2 data. For the PALSAR data, they are clustered in $\mathrm{Z1}$ and $\mathrm{Z} 2$. We observe that both datasets show a majorly dominant even-bounce scattering power component in the urban area usually characterized by dihedral targets. However, for the RS-2 data, the Z7 cluster indicates dominant odd-bounce scattering power. After analyzing the combinations of $H, A$, we noticed that $H A$ is high for the RS-2 data, suggesting that there might exist two mechanisms with the same probability. Due to this reason, the percentage of pixels in $\mathrm{Z} 1(49.7 \%)$ is comparable to $\mathrm{Z7}(33.4 \%)$.

On the other hand, depending on some buildings' orientation within the resolution cell, $P_{c}$ power dominates over $P_{v}$. Hence, a marginal percentage of pixels in the $\mathrm{Z} 2$ region is also evident over the urban area. Concerning the ALOS PALSAR data, the value of $(1-H) A$ is high, indicating two scattering mechanisms with a dominant process. Hence, we observe only $\mathrm{Z} 1$ and $\mathrm{Z} 2$ clusters for this data set. Besides, for both datasets, $\alpha_{s}^{1} \approx 60^{\circ}$, indicating it as majorly a dihedral scatterer.

TABLE VI

PERCENTAGE OF PIXELS IN DIFFERENT CLUSTERS OVER ORIENTED URBAN USING RS-2 AND ALOS PALSAR DATA (Z3: $P_{d}>P_{v}>P_{s}>P_{c}$; Z4: $P_{d}>P_{v}>P_{c}>P_{s} ; \mathrm{Z7}: P_{s}>P_{d}>P_{v}>P_{c}$; Z13: $P_{v}>P_{s}>P_{d}>P_{c}$; Z14: $P_{v}>P_{s}>P_{c}>P_{d}$; Z15: $\left.P_{v}>P_{d}>P_{s}>P_{c} ; \mathrm{Z} 18: P_{v}>P_{c}>P_{d}>P_{s}\right)$

\begin{tabular}{|c|c|c|c|}
\hline \multicolumn{2}{|r|}{ RS-2 } & \multicolumn{2}{|c|}{ ALOS PALSAR } \\
\hline Class & Percent $(\%)$ & Class & Percent $(\%)$ \\
\hline $\mathrm{Z} 3$ & 0.00 & $\mathrm{Z} 3$ & 43.10 \\
\hline Z4 & 29.60 & Z4 & 0.00 \\
\hline $\mathrm{Z7}$ & 12.43 & $\mathrm{Z7}$ & 0.00 \\
\hline Z13 & 40.17 & Z13 & 20.46 \\
\hline Z14 & 11.86 & Z14 & 17.60 \\
\hline Z15 & 0.00 & Z15 & 15.86 \\
\hline Z18 & 5.94 & Z18 & 2.99 \\
\hline
\end{tabular}

Table VI shows that there are several clustering zones over the oriented urban area (OU). The orientation of the urban area about the radar line of sight suggests randomness in the scattered electromagnetic wave. Therefore, we observe different scattering mechanisms in this area. The value of $H(1-A)$ is higher than any other combinations of $H$ and $A$ for both datasets, which indicates a random scattering process within the resolution cell. For the RS- 2 data, the total percentage of pixels in Z13 and Z14 is high due to dominant diffused scattering, evident from Figure 12 


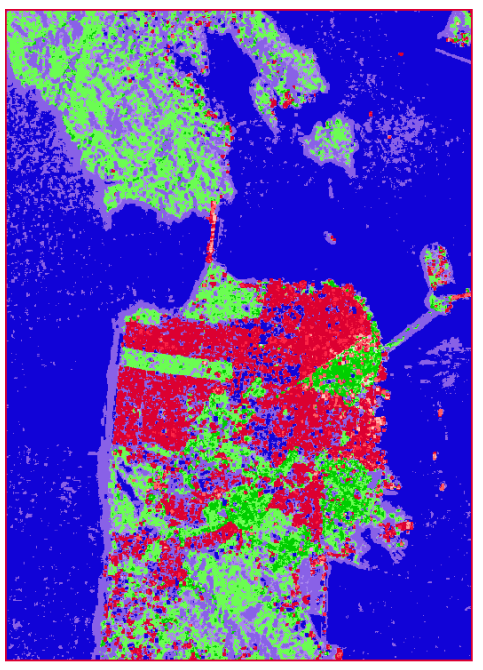

(a) RS-2

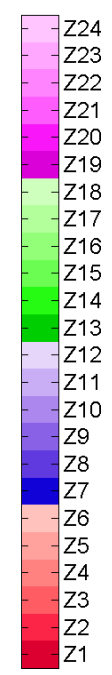

Z1

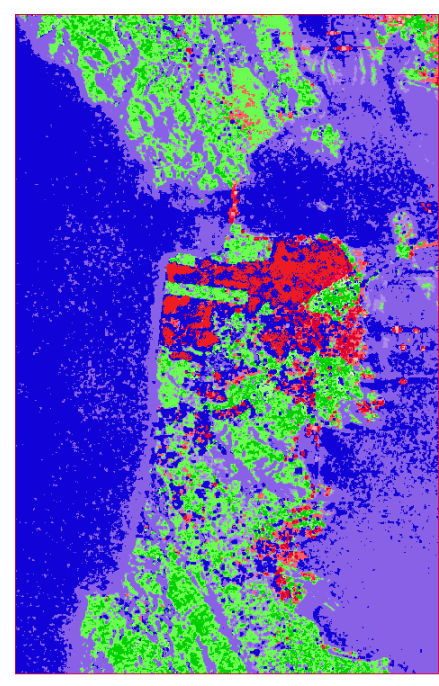

(b) ALOS PALSAR

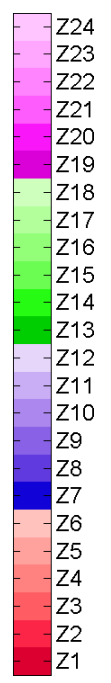

Fig. 15. Unsupervised clusters over different scattering targets using RS-2, ALOS PALSAR and TerraSAR-X data.

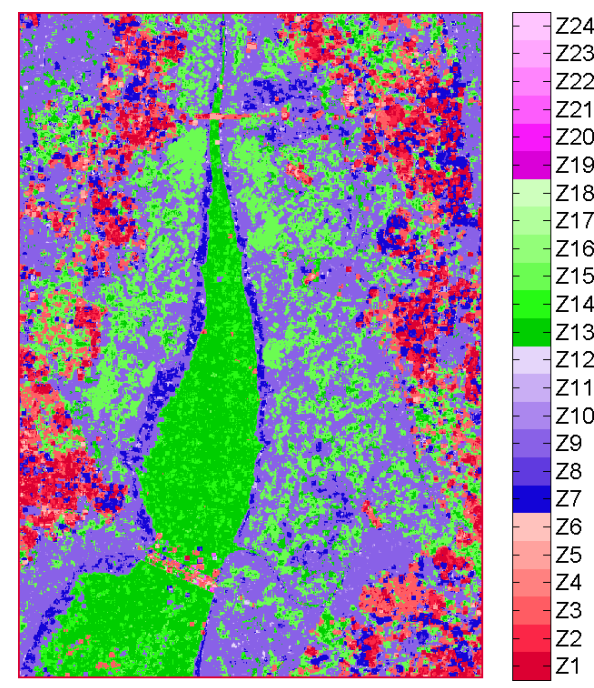

(c) TerraSAR-X
However, oriented structures generated a significant amount of helix scattering power due to which, for a few circumstances, it dominates over the odd-bounce power component. Therefore we observe the Z4 cluster in the scene. For a few other pixels, the helix power component is almost comparable to the even-bounce scattering power component, which shows the appearance of the Z18 cluster over the oriented urban area.

For the ALOS PALSAR data, the percentage of pixels in the Z3 cluster $(43.10 \%)$ is higher than any other clusters due to the dominant even-bounce scattering. This mechanism might be because of the difference in the wavelengths, which suggests high penetration ability compared to the RS- 2 data.

Multiple scattering phenomena increased the values of $P_{v}$ more than that measured in the orthogonal urban area. Therefore, the clusters Z13, Z14, Z15, and Z18 are evident in Table VI. Alongside this, a high amount of $P_{c}$ power sometimes makes it the second or the third dominant depending on the significance of oriented buildings. Over OU, $\alpha_{s}^{1} \approx 46^{\circ}$ for both datasets indicate the scatterer behaviour towards the dihedral type accompanied by multiple scattering phenomena.

TABLE VII

PERCENTAGE OF PIXELS IN DIFFERENT CLUSTERS OVER VEGETATION USING RS-2 AND ALOS PALSAR DATA (Z9: $P_{s}>P_{v}>P_{d}>P_{c}$; Z10: $P_{s}>P_{v}>P_{c}>P_{d}$; Z13: $P_{v}>P_{s}>P_{d}>P_{c}$; Z15: $\left.P_{v}>P_{d}>P_{s}>P_{c}\right)$

\begin{tabular}{|c|c|c|c|}
\hline \multicolumn{2}{|r|}{ RS-2 } & \multicolumn{2}{|c|}{ ALOS PALSAR } \\
\hline Class & Percent $(\%)$ & Class & Percent (\%) \\
\hline Z9 & 0.00 & Z9 & 6.64 \\
\hline Z10 & 8.46 & Z10 & 9.96 \\
\hline Z13 & 25.93 & Z13 & 0.00 \\
\hline Z15 & 65.61 & Z15 & 83.39 \\
\hline
\end{tabular}

Table VII shows that, over the vegetation area, pixels in the Z10, Z13 and Z15 clusters are evident in the RS-2 data, while pixels in Z9, Z10 and Z15 clusters are evident in the ALOS PALSAR data. We observe that, for both datasets, the diffused power scattering component is the first dominant. In this case, scattering randomness is primarily due to the geometry and composition of branches and twigs and their multiple interactions with wavelengths of comparable dimension. The second dominant $P_{d}$ power in Z15 is likely due to the interaction of the EM wave with the ground and the vegetation trunk.

\section{TABLE VIII}

PERCENTAGE OF PIXELS IN DIFFERENT CLUSTERS OVER MANGROVE AND URBAN USING TERRASAR-X DATA (Z1: $P_{d}>P_{s}>P_{v}>P_{c}$. Z2: $P_{d}>P_{s}>P_{c}>P_{v} ; \mathrm{Z7}: P_{s}>P_{d}>P_{v}>P_{c}$ Z9: $P_{s}>P_{v}>P_{d}>P_{c} ; \mathrm{Z} 10: P_{s}>P_{v}>P_{c}>P_{d}$; Z13: $P_{v}>P_{s}>P_{d}>P_{c}$; Z15: $\left.P_{v}>P_{d}>P_{s}>P_{c}\right)$

\begin{tabular}{|c|c|c|c|}
\hline \multicolumn{2}{|c|}{ Urban } & \multicolumn{2}{|c|}{ Mangrove } \\
\hline Class & Percent $(\%)$ & Class & Percent $(\%)$ \\
\hline Z1 & 38.30 & Z1 & 0.00 \\
\hline $\mathrm{Z} 2$ & 49.10 & $\mathrm{Z} 2$ & 0.00 \\
\hline $\mathrm{Z7}$ & 12.60 & $\mathrm{Z7}$ & 0.00 \\
\hline Z9 & 0.00 & Z9 & 15.60 \\
\hline Z13 & 0.00 & Z13 & 13.30 \\
\hline Z15 & 0.00 & Z15 & 71.10 \\
\hline
\end{tabular}

Besides, for those pixels, the amount of $P_{s}$ power is less due to the minimum direct interaction of the EM wave with only leaves and foliage. Also, the amount of $P_{c}$ power in those pixels is low due to higher symmetric scattering condition. Therefore, the $P_{s}$ power component becomes the third dominant, while the $P_{c}$ power component is the fourth dominant.

On the other hand, for some pixels, the $P_{s}$ power dominates over $P_{d}$ and $P_{v}$ powers, which might be due to high leaf area content in those pixels. This increased amount of leaf area has produced more $P_{s}$ power due to which Z9 and Z10 clusters are observed in the PALSAR and RS-2 data, respectively. However, based on the difference between the wavelengths and the depth of penetration, the amount of $P_{c}$ power varies from RS-2 to PALSAR datasets. Therefore, the dominance in the $P_{c}$ power component changes from RS-2 to PALSAR 
datasets.

The variations of clusters over urban and mangrove areas using the TerraSAR-X data are shown in Table VIII. Like RS-2 data, pixels in the urban area (U) are clustered in Z1, $\mathrm{Z} 2$ and $\mathrm{Z7}$. Therefore, the high even-bounce scattering power component characterizes the target similar to the dihedral. However, due to the small wavelength of X-band, the $\mathrm{Z7}$ cluster is evident, which is also indicated by the combined analysis of $H$ and $A$. On the other hand, the mangrove region (M) shows a dominant $P_{v}$ scattering power. However, it should be noted that mangrove thrives abundantly in brackish water areas, e.g., estuaries and mud-laden rivers. These areas comprising a shallow water column can generate a sufficient amount of even-bounce and diffused scattering mechanism. Hence, the appearance of the Z15 cluster in the appropriate proportion is evident for this area. On the other hand, the top canopy layer and the leaves generated an odd-bounce scattering mechanism, due to which the Z9 and Z13 clusters are also apparent within this area.

\section{Conclusions}

This study proposed a model-free four-component scattering power decomposition technique for full polarimetric Synthetic Aperture Radar (SAR) data. This technique is an extension of our model-free three-component decomposition technique. The introduction of an asymmetry parameter resolves the ambiguity between the even-bounce and the helix scattering mechanisms. This scattering asymmetry component helps to characterize scattering from human-made structures in urban areas. Therefore, the scattering asymmetry component might infer unique polarimetric properties about a target present in the scene.

Therefore, we have included the $\tau_{\mathrm{FP}}$ parameter along with $\theta_{\mathrm{FP}}$ to capture the scattering asymmetry from a target. Utilizing these two parameters simultaneously, we proposed the four scattering power components: even-bounce $\left(P_{d}\right)$, odd-bounce $\left(P_{s}\right)$, diffused $\left(P_{v}\right)$ and helix $\left(P_{c}\right)$.

Unlike conventional model-based decompositions, we do not specify a priori, canonical scattering models, to derive the power components. Our technique does not produce any negative power pixels, which is a significant drawback in most model-based approaches. In this regard, our approach explicitly included the amount of polarized scattered information in terms of the Barakat degree of polarization.

Most importantly, the proposed technique enumerates the power components simultaneously, which reduces the computational complexity of the problem.

Moreover, it also enhances interpretability by avoiding the intricate branching criteria of model-based decompositions. All the characterization parameters (i.e., $\theta_{\mathrm{FP}}$ and $\tau_{\mathrm{FP}}$ ), and the scattering power components are also roll-invariant.

Results show that the proposed technique performs reasonably well over diverse landcover classes compared to several methods: Yamaguchi 4-component decomposition with rotation (Y4R), General four-component scattering power decomposition (G4U), Adaptive General Four-Component Scattering Power Decomposition (AG4U), and Six component scattering power decomposition (i6SD). The polarized power components over the ocean and the urban areas are enhanced. Moreover, due to the unique roll-invariant nature, the $P_{d}$ power component has also increased relative to $\mathrm{Y} 4 \mathrm{R}, \mathrm{G} 4 \mathrm{U}$, AG4U and i6SD.

Besides, the ground-trunk and the ground-branch interactions over vegetation areas are evident in our study due to which specific polarized power components have increased over these areas. The introduction of the $P_{c}$ power component has provided detailed information about scattering asymmetry over various targets. Over ocean and vegetation surfaces, the $P_{c}$ power exhibits minimal values due to the scattering symmetry property. Contrarily, the $P_{c}$ power values have increased for urban areas. Nonetheless, over oriented urban areas, the $P_{c}$ power component is significant due to high scattering asymmetry.

The proposed unsupervised clustering technique can adequately capture target scattering variations based on dominant scattering mechanisms. Variations of clusters from one target to another are noticeable from the results obtained using images acquired by three different frequencies (viz., $\mathrm{C}-$, L-, and X-bands). Moreover, the classes derived from this clustering technique are good representative and provide enhanced insight into targets' scattering mechanisms based on their physical properties. Thus the proposed model-free decomposition technique and the unsupervised clustering technique possess an excellent potential for land cover analysis using FP SAR data.

\section{REPRODUCIBILITY AND REPLICABILITY}

Following the guidelines presented in Ref. [29], we made available the code that supports the reproducibility and replicability of this work in a Github repository: https://github.com/ Subho07/MF4CF [30] .

\section{ACKNOWLEDGEMENT}

The authors want to thank the anonymous reviewers for their valuable comments and suggestions that have helped to enhance the technical content of the manuscript. The authors are thankful to the German Aerospace Center (DLR) for providing the TerraSAR-X data under the Announcement of Opportunity (AO) proposal ID: TanDEM-X CalVal 6884. This work was partially supported by CNPq.

\section{APPENDIX}

\section{GEOMETRICAL INTERPRETATION OF $\theta_{\mathrm{FP}}$}

We derive all the auxiliary variables in terms of the elements of the coherency matrix $\mathbf{T}$ which is then equivalently represented in terms of the elements of the Kennaught matrix $\mathbf{K}$. The total power is $\mathrm{TP}=T_{11}+T_{22}+T_{33}=2 K_{11}$, where $T_{11}, T_{22}$, and $T_{33}$ are the elements of $\mathbf{T}$, and $K_{11}$ is the $(1,1)$ element of $\mathbf{K}$. Using these elements, the geometrical description of $\theta_{\mathrm{FP}}$ is shown in Fig. 16, where $\overline{\mathrm{OQ}}$ represents TP which can be decomposed into polarized and unpolarized components as

$$
\mathrm{TP}=\underbrace{m_{\mathrm{FP}} \mathrm{TP}}_{\text {Polarized part }}+\underbrace{\left(1-m_{\mathrm{FP}}\right) \mathrm{TP}}_{\text {Unpolarized part }}
$$




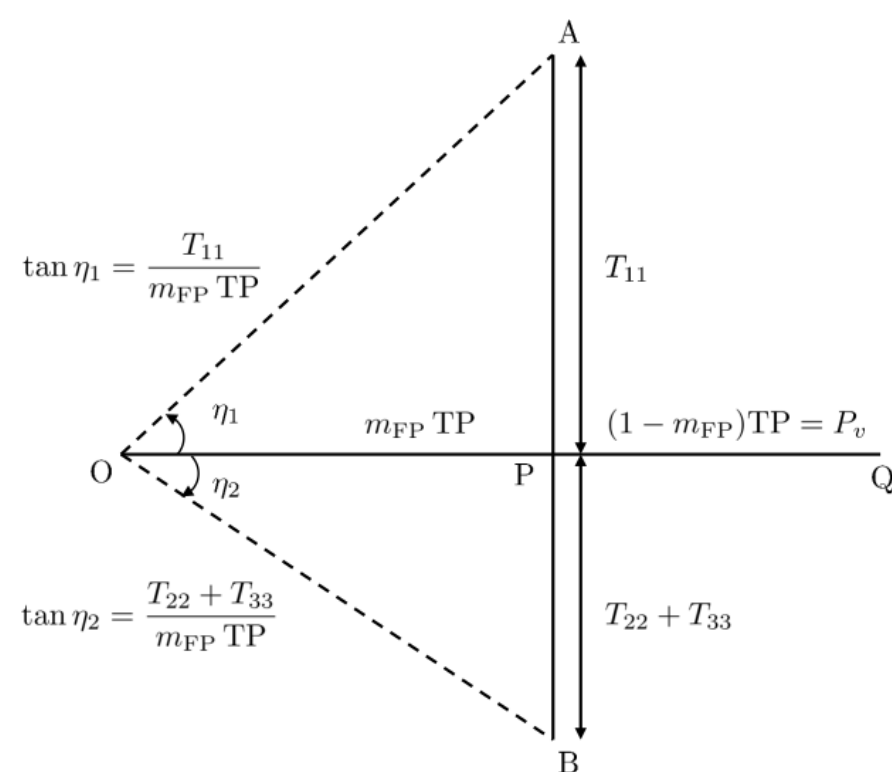

Fig. 16. Geometrical representation of $\theta_{\text {FP }}$

In the figure, $\overline{\mathrm{OP}}$ represents the polarized part of the total power, while $\overline{\mathrm{PQ}}$ represents the depolarized part. The point $\mathrm{P}$ discriminates the polarized component from the unpolarized component. We consider an arbitrary projection of $T_{11}$ and $T_{22}+T_{33}$ on $\overline{\mathrm{OP}} ; \overline{\mathrm{OA}}$ makes an angle $\eta_{1}$ with $\overline{\mathrm{OP}}$, and $\overline{\mathrm{OB}}$ makes an angle $\eta_{2}$ with $\overline{\mathrm{OP}}$. Therefore, with these projections, we are primarily interested in finding the proportion of regular and irregular components of the scattering wave to the total polarized power:

$$
\tan \eta_{1}=\frac{T_{11}}{m_{\mathrm{FP}} \mathrm{TP}}, \quad \tan \eta_{2}=\frac{T_{22}+T_{33}}{m_{\mathrm{FP}} \mathrm{TP}}
$$

With the following relationships between elements of $\mathbf{T}$ and $\mathbf{K}$ we can write

$$
\begin{aligned}
& \eta_{1}=\tan ^{-1} \frac{T_{11}}{m_{\mathrm{FP}} \mathrm{TP}}=\tan ^{-1} \frac{K_{11}-K_{44}}{2 m_{\mathrm{FP}} K_{11}}, \\
& \eta_{2}=\tan ^{-1} \frac{T_{22}+T_{33}}{m_{\mathrm{FP}} \mathrm{TP}}=\tan ^{-1} \frac{K_{11}+K_{44}}{2 m_{\mathrm{FP}} K_{11}},
\end{aligned}
$$

where, $K_{11}=\left(T_{11}+T_{22}+T_{33}\right) / 2$ and $K_{44}=\left(T_{22}+\right.$ $\left.T_{33}-T_{11}\right) / 2$. Therefore, we can write, $T_{11}=K_{11}-K_{44}$ and $T_{22}+T_{33}=K_{11}+K_{44}$. The difference between $\eta_{1}$ and $\eta_{2}$ essentially characterizes scattering from a target denoted by $\theta_{\mathrm{FP}}$ and defined as

$$
\begin{aligned}
\tan \theta_{\mathrm{FP}} & =\tan \left(\eta_{1}-\eta_{2}\right) \\
& =\frac{4 m_{\mathrm{FP}} K_{11} K_{44}}{K_{44}^{2}-\left(1+4 m_{\mathrm{FP}}^{2}\right) K_{11}^{2}} .
\end{aligned}
$$

\section{REFERENCES}

[1] A. Freeman and S. L. Durden, "A three-component scattering model for polarimetric SAR data," IEEE Trans. Geosci. Remote Sens., vol. 36, no. 3, pp. 963-973, 1998.

[2] J.-S. Lee, M. R. Grunes, T. L. Ainsworth, L.-J. Du, D. L. Schuler, and S. R. Cloude, "Unsupervised classification using polarimetric decomposition and the complex Wishart classifier," IEEE Trans. Geosci. Remote Sens., vol. 37, no. 5, pp. 2249-2258, 1999.
[3] J.-S. Lee, M. R. Grunes, E. Pottier, and L. Ferro-Famil, "Unsupervised terrain classification preserving polarimetric scattering characteristics," IEEE Trans. Geosci. Remote Sens., vol. 42, no. 4, pp. 722-731, 2004.

[4] Y. Yamaguchi, T. Moriyama, M. Ishido, and H. Yamada, "Fourcomponent scattering model for polarimetric SAR image decomposition," IEEE Trans. Geosci. Remote Sens., vol. 43, no. 8, pp. 1699-1706, 2005.

[5] M. Arii, J. J. van Zyl, and Y. Kim, "A general characterization for polarimetric scattering from vegetation canopies," IEEE Trans. Geosci. Remote Sens., vol. 48, no. 9, pp. 3349-3357, 2010.

[6] J. J. Van Zyl, M. Arii, and Y. Kim, "Model-based decomposition of polarimetric SAR covariance matrices constrained for nonnegative eigenvalues," IEEE Trans. Geosci. Remote Sens., vol. 49, no. 9, pp. 3452-3459, 2011.

[7] Y. Cui, Y. Yamaguchi, J. Yang, H. Kobayashi, S.-E. Park, and G. Singh, "On complete model-based decomposition of polarimetric SAR coherency matrix data," IEEE Trans. Geosci. Remote Sens., vol. 52, no. 4, pp. 1991-2001, 2013.

[8] W. An, Y. Cui, and J. Yang, "Three-component model-based decomposition for polarimetric SAR data," IEEE Trans. Geosci. Remote Sens. vol. 48, no. 6, pp. 2732-2739, 2010.

[9] J.-S. Lee and T. L. Ainsworth, "The effect of orientation angle compensation on coherency matrix and polarimetric target decompositions," IEEE Trans. Geosci. Remote Sens., vol. 49, no. 1, pp. 53-64, 2010.

[10] Y. Yamaguchi, A. Sato, W.-M. Boerner, R. Sato, and H. Yamada, "Fourcomponent scattering power decomposition with rotation of coherency matrix," IEEE Trans. Geosci. Remote Sens., vol. 49, no. 6, pp. 22512258, 2011.

[11] G. Singh, Y. Yamaguchi, and S.-E. Park, "General four-component scattering power decomposition with unitary transformation of coherency matrix," IEEE Trans. Geosci. Remote Sens., vol. 51, no. 5, pp. 3014 3022, May 2013.

[12] Z. Shuang, Y. Xiangchuan, and W. Lu, "Modified version of threecomponent model-based decomposition for polarimetric SAR data," $J$. Syst. Eng. Electron., vol. 30, no. 2, pp. 270-277, 2019.

[13] S.-W. Chen, X.-S. Wang, S.-P. Xiao, and M. Sato, "General polarimetric model-based decomposition for coherency matrix," IEEE Trans. Geosci. Remote Sens., vol. 52, no. 3, pp. 1843-1855, 2013.

[14] A. Bhattacharya, G. Singh, S. Manickam, and Y. Yamaguchi, "An adaptive general four-component scattering power decomposition with unitary transformation of coherency matrix (AG4U)," IEEE Geosci. Remote Sens. Lett., vol. 12, no. 10, pp. 2110-2114, 2015.

[15] A. Bhattacharya, A. Muhuri, S. De, S. Manickam, and A. C. Frery, "Modifying the Yamaguchi four-component decomposition scattering powers using a stochastic distance," IEEE J. Sel. Topics Appl. Earth Observ. Remote Sens., vol. 8, no. 7, pp. 3497-3506, July 2015.

[16] D. Ratha, E. Pottier, A. Bhattacharya, and A. C. Frery, "A polsar scattering power factorization framework and novel roll-invariant parameterbased unsupervised classification scheme using a geodesic distance," IEEE Trans. Geosci. Remote Sens., pp. 1-17, 2019.

[17] G. Singh and Y. Yamaguchi, "Model-based six-component scattering matrix power decomposition," IEEE Trans. Geosci. Remote Sens., vol. 56, no. 10, pp. 5687-5704, 2018.

[18] G. Singh, R. Malik, S. Mohanty, V. S. Rathore, K. Yamada, M. Umemura, and Y. Yamaguchi, "Seven-component scattering power decomposition of POLSAR coherency matrix," IEEE Trans. Geosci. Remote Sens., vol. 57, no. 11, pp. 8371-8382, 2019.

[19] S. Dey, A. Bhattacharya, D. Ratha, D. Mandal, and A. C. Frery, "Target characterization and scattering power decomposition for full and compact polarimetric SAR data," IEEE Trans. Geosci. Remote Sens., pp. $1-18,2020$.

[20] R. Barakat, "n-fold polarization measures and associated thermodynamic entropy of N partially coherent pencils of radiation," Opt. Acta, vol. 30, no. 8, pp. 1171-1182, 1983.

[21] J. R. Huynen, "Phenomenological theory of radar targets," PhD dissertation, Technical Univ., Delf, The Netherlands, 1970.

[22] R. Touzi, "Target scattering decomposition in terms of roll-invariant target parameters," IEEE Trans. Geosci. Remote Sens., vol. 45, no. 1, pp. 73-84, 2006.

[23] P. Réfrégier, F. Goudail, P. Chavel, and A. Friberg, "Entropy of partially polarized light and application to statistical processing techniques," $J$. Opt. Soc. Amer. A, vol. 21, no. 11, pp. 2124-2134, 2004.

[24] R. Barakat, "Degree of polarization and the principal idempotents of the coherency matrix," Opt. Commun., vol. 23, no. 2, pp. 147-150, 1977.

[25] J.-S. Lee and E. Pottier, Polarimetric radar imaging: from basics to applications. CRC press, 2017. 
[26] F. M. Henderson and A. J. Lewis, Principles and Applications of Imaging Radar: Manual of Remote Sensing, 3rd ed. John Wiley and Sons, Inc., Somerset, NJ (United States), 1998, vol. 2.

[27] G. Singh, Y. Yamaguchi, and S.-E. Park, "General four-component scattering power decomposition with unitary transformation of coherency matrix," IEEE Transactions on Geoscience and Remote Sensing, vol. 51, no. 5, pp. 3014-3022, 2012.

[28] T. Ainsworth, D. Schuler, and J.-S. Lee, "Polarimetric SAR characterization of man-made structures in urban areas using normalized circularpol correlation coefficients," Remote Sens. Environ., vol. 112, no. 6, pp. 2876-2885, 2008.

[29] A. C. Frery, L. Gomez, and A. C. Medeiros, "A badging system for reproducibility and replicability in remote sensing research," IEEE $J$. Sel. Topics Appl. Earth Observ. Remote Sens., vol. 13, pp. 4988-4995, 2020.

[30] N. Bhogapurapu, S. Dey, D. Mandal, A. Bhattacharya, and Y. S. Rao, "PolSAR tools: A QGIS plugin for generating SAR descriptors," Journal of Open Source Software, 2021.

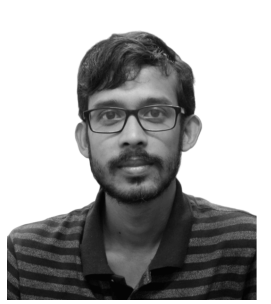

Subhadip Dey (S'17) received the B.Tech degree in Agricultural Engineering from Bidhan Chandra Krishi Viswavidyalaya, India. He received the M. Tech degree in Aquacultural Engineering, Department of Agricultural and Food Engineering, Indian Institute of Technology Kharagpur, India. He is currently pursuing the Ph.D. degree at the Microwave Remote Sensing Lab, Centre of Studies in Resources Engineering (CSRE), Indian Institute of Technology Bombay, India. His current research interests are land cover classification and agricultural crop mapping and monitoring using Synthetic Aperture Radar data.

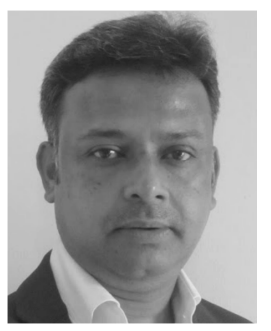

Avik Bhattacharya (M'08-SM'16) received the integrated M.Sc. degree in Mathematics from the Indian Institute of Technology, Kharagpur, India, in 2000 and the Ph.D. degree in remote sensing image processing and analysis from Télécom ParisTech, Paris, France, and the Ariana Research Group, Institut National de Recherche en Informatique et en Automatique (INRIA), Sophia Antipolis, Nice, France, in 2007. He is currently an Associate Professor at the Centre of Studies in Resources Engineering, Indian Institute of Technology Bombay (CSRE, IITB), Mumbai, India. Before joining IITB, he was a Canadian Government Research Fellow at the Canadian Centre for Remote Sensing (CCRS) in Ottawa, ON, Canada. He received the Natural Sciences and Engineering Research Council of Canada visiting scientist fellowship at the Canadian national laboratories, from 2008 to 2011. His current research interests include SAR polarimetry, statistical analysis of polarimetric SAR images, applications of Radar Remote Sensing in Agriculture, Cryosphere, Urban and Planetary studies. Dr. Bhattacharya is the Editor-in-Chief of IEEE Geoscience and Remote Sensing Letters. He was an Associate Editor of IEEE GRSL. He has been the Guest Editor of the special issue on Applied Earth Observations and Remote Sensing in India in IEEE Journal of Selected Topics in Applied Earth Observations and Remote Sensing (J-STARS), 2017. He was one of the guest editors of the special stream on Advanced Statistical Techniques in SAR Image Processing and Analysis in IEEE Geoscience and Remote Sensing LetterS, 2018. He is the Founding Chairperson of the IEEE Geoscience and Remote Sensing Society (GRSS) Chapter of the Bombay Section. He is currently leading the Microwave Remote Sensing Lab (www.mrslab.in) at CSRE, IITB.

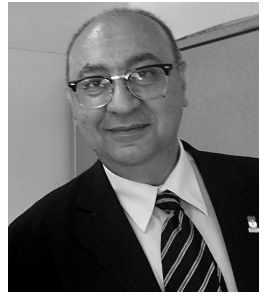

Alejandro C. Frery (S'92-SM'03) received the B.Sc. degree in Electronic and Electrical Engineering from the Universidad de Mendoza, Mendoza, Argentina. His M.Sc. degree was in Applied Mathematics (Statistics) from the Instituto de Matemática Pura e Aplicada (IMPA, Rio de Janeiro) and his Ph.D. degree was in Applied Computing from the Instituto Nacional de Pesquisas Espaciais (INPE, São José dos Campos, Brazil). He was the founder of LaCCAN - Laboratório de Computação Científica e Análise Numérica, Universidade Federal de Alagoas, Maceió, Brazil, and holds a Huashan Scholar position (2019-2021) with the Key Lab of Intelligent Perception and Image Understanding of the Ministry of Education, Xidian University, Xi'an, China. He was the Editor-in-Chief of IEEE Geoscience and Remote Sensing Letters (2014-2018). In 2018 he received the IEEE GRSS Regional Leader Award. His research interests are statistical computing and stochastic modeling.

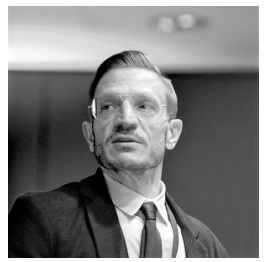

Carlos López-Martínez (S'97-M'04-SM'11) received the MSc. degree in electrical engineering and the Ph.D. degree from the Universitat Politècnica de Catalunya, Barcelona, Spain, in 1999 and 2003, respectively. Dr. López-Martínez is Associate Professor in the area of remote sensing and microwave technology in the Universitat Politècnica de Catalunya, Barcelona, Spain. He has a large professional international experience at DLR (Germany), at the University of Rennes 1 (France), and as a group leader of the Remote Sensing and Natural Resources Modelling team in the Luxembourg Institute of Science and Technology (Luxembourg). His research interests include Synthetic Aperture Radar (SAR) theory, statistics and applications, multidimensional SAR, radar polarimetry, physical parameter inversion, advanced digital signal processing, estimation theory, and harmonic analysis. Dr. López-Martínez has authored more than 200 articles in journals, books, and conference proceedings, and received the EUSAR 2002 Conference Student Prize Paper Award, coauthored the paper awarded with the EUSAR 2012 Conference First Place Student Paper Award, and received the IEEE-GRSS 2013 GOLD Early Career Award. Dr. López-Martínez has broad academic teaching experience from bachelor, master, and $\mathrm{PhD}$ levels to advanced technical tutorials presented at international conferences and space and research institutions worldwide. He is an associate editor of the IEEE-JSTARS journal and the MDPI Remote Sensing, acting also as invited guest editor for several special issues. He has collaborated in the Spanish PAZ and the ESA's SAOCOM-CS missions, in the proposal of the Parsifal mission and he is member of the ESA's Sentinel ROSE-L Mission Advisory Group. He was appointed vice-president of the IEEE-GRSS Spanish chapter, and in 2016 he became its secretary and treasurer. From 2011, Dr. López-Martínez collaborates with the IEEEGRSS Globalization initiative in Latin America, contributing to the creation of the IEEE-GRSS Chilean chapter and the organization of the 2020 LAGIRSS conference, being appointed as Latin America liaison in 2019. He is also co-chair of the Tutorial Technical Committee of the Indian 2020 InGARSS conference. 


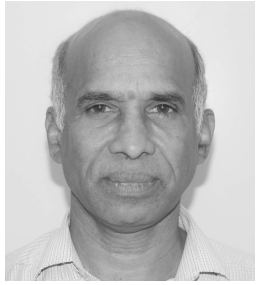

Y. S. Rao received the M.Sc. degree in physics from Andhra University, India, in 1982, and the Ph.D. degree in passive microwave remote sensing of soil moisture from the Indian Institute of Technology (IIT) Bombay, Mumbai, in 1992. He joined the Centre of Studies Resources Engineering, IIT Bombay, in 1985, as a Senior Research Assistant and, then became a Research Scientist in 1999. During 2005-2009, he was a Senior Research Scientist and, then Associate Professor from 2009 to 2014. He is currently continuing as a Professor. He worked in both passive and active microwave remote sensing for several applications viz. soil moisture, flood mapping, and land use/land cover. He has participated in several spaceborne campaigns for collecting synchronous ground-truth data and has experience in handling various datasets for several applications. His research interests include application of polarimetry for geo-physical parameter retrieval and SAR interferometry for DEM and displacement map generation. 